ARTICLE

\title{
The genome-wide impact of trisomy 21 on DNA methylation and its implications for hematopoiesis
}

Ivo S. Muskens 1,2, Shaobo Li (10) 1,2,13, Thomas Jackson3,13, Natalina Elliot ${ }^{3,13}$, Helen M. Hansen4, Swe Swe Myint ${ }^{1,2}$, Priyatama Pandey ${ }^{1,2}$, Jeremy M. Schraw ${ }^{5,6}$, Ritu Roy ${ }^{7}$, Joaquin Anguiano ${ }^{4}$, Katerina Goudevenou ${ }^{3}$, Kimberly D. Siegmund (1) ${ }^{8}$, Philip J. Lupo ${ }^{5,6}$, Marella F. T. R. de Bruijn (D) ${ }^{9}$, Kyle M. Walsh (10 10,11, Paresh Vyas (10) ${ }^{9}$, Xiaomei Ma ${ }^{12}$, Anindita Roy (1) ${ }^{3}$, Irene Roberts ${ }^{3}$, Joseph L. Wiemels ${ }^{1,2} \&$ Adam J. de Smith (1) 1,2四

Down syndrome is associated with genome-wide perturbation of gene expression, which may be mediated by epigenetic changes. We perform an epigenome-wide association study on neonatal bloodspots comparing 196 newborns with Down syndrome and 439 newborns without Down syndrome, adjusting for cell-type heterogeneity, which identifies 652 epigenome-wide significant $\mathrm{CpGs}\left(P<7.67 \times 10^{-8}\right)$ and 1,052 differentially methylated regions. Differential methylation at promoter/enhancer regions correlates with gene expression changes in Down syndrome versus non-Down syndrome fetal liver hematopoietic stem/progenitor cells $(P<0.0001)$. The top two differentially methylated regions overlap RUNX1 and FLI1, both important regulators of megakaryopoiesis and hematopoietic development, with significant hypermethylation at promoter regions of these two genes. Excluding Down syndrome newborns harboring preleukemic GATA1 mutations $(N=30)$, identified by targeted sequencing, has minimal impact on the epigenome-wide association study results. Down syndrome has profound, genome-wide effects on DNA methylation in hematopoietic cells in early life, which may contribute to the high frequency of hematological problems, including leukemia, in children with Down syndrome.

\footnotetext{
${ }^{1}$ Center for Genetic Epidemiology, Department of Preventive Medicine, Keck School of Medicine of the University of Southern California, Los Angeles, CA, USA. ${ }^{2}$ Norris Comprehensive Cancer Center, University of Southern California, Los Angeles, USA. ${ }^{3}$ Department of Paediatrics and MRC Molecular Haematology Unit, Weatherall Institute of Molecular Medicine, Oxford University and BRC Blood Theme, NIHR Oxford Biomedical Centre, Oxford, UK. ${ }^{4}$ Department of Neurological Surgery, University of California San Francisco, San Francisco, CA, USA. ${ }^{5}$ Department of Pediatrics, Section of HematologyOncology, Baylor College of Medicine, Houston, TX, USA. ${ }^{6}$ Texas Children's Cancer and Hematology Centers, Texas Children's Hospital, Houston, TX, USA.

${ }^{7}$ Computational Biology and Informatics, University of California San Francisco, San Francisco, CA, USA. ${ }^{8}$ Department of Preventive Medicine, Keck School of Medicine of the University of Southern California, Los Angeles, CA, USA. ${ }^{9}$ MRC Molecular Haematology Unit, MRC Weatherall Institute of Molecular Medicine, University of Oxford, Oxford, UK. ${ }^{10}$ Department of Neurosurgery, Duke University, Durham, NC, USA. ${ }^{11}$ Department of Pediatrics, Duke University, Durham, NC, USA. ${ }^{12}$ Department of Chronic Disease Epidemiology, Yale School of Public Health, New Haven, CT, USA. ${ }^{13}$ These authors contributed equally:

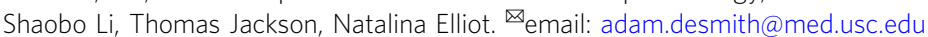


D own syndrome (DS), caused by constitutive trisomy of chromosome 21 (T21), is one of the most common genetic disorders $^{1}$, and is associated with a spectrum of adverse phenotypes $^{2}$. DS is characterized by defects in immune system development and in hematopoiesis, with DS fetuses having perturbed megakaryocyte/red cell and B-lymphoid development ${ }^{3}$ and DS children having a higher frequency of lymphopenia ${ }^{4}$ and infections ${ }^{5}$. Furthermore, children with DS have a 20-30-fold increased risk of acute lymphoblastic leukemia (ALL) and a 500fold increased risk of acute megakaryoblastic leukemia (AMKL), while displaying a decreased risk of common adult-onset solid tumors $^{6,7}$. Approximately $10 \%$ of DS newborns present with transient abnormal myelopoiesis (TAM), a preleukemic disorder associated with increased peripheral blood blast cells and pathognomonic somatic mutations in the X-linked erythro-megakaryocytic transcription factor (TF) gene GATA1 ${ }^{8}$. A further $15-20 \%$ have acquired GATA1 mutations without clinical features, so-called "Silent $\mathrm{TAM}^{8}$." TAM and Silent TAM resolve spontaneously in most cases, but up to $20 \%$ acquire additional oncogenic mutations and develop frank AMKL ${ }^{9,10}$.

DS-related phenotypes vary greatly in presentation and penetrance 2,11 , and understanding the biological basis of that variation may highlight novel therapeutic approaches, and shed light on the etiology of these conditions in non-DS individuals ${ }^{11}$. Altered expression of genes, both on Hsa21 and genome-wide, is widely accepted to play a key role in the manifestation of DS-related phenotypes, many of which originate prenatally ${ }^{3}$. Several studies, including in monozygotic twins discordant for T21, provide strong evidence for the effect of T21 on the human transcriptome ${ }^{12,13}$, with substantial interindividual variability in expression patterns ${ }^{14}$.

Studying baseline epigenetic effects of T21 at birth is a powerful approach to pinpoint broad epigenetic landscapes and/or individual genes that underlie DS-related phenotypes. Nevertheless, comprehensive analysis of genome-wide DNA methylation changes in DS is lacking; previous studies comprised very few $(N<30)$ individuals, did not explore interethnic differences, nor account for the potential impact of somatic GATA1 mutationharboring clones ${ }^{15-19}$.

Here, we investigate T21-associated changes in DNA methylation among 196 DS and 439 non-DS newborn blood samples, and consider the potential confounding effects of somatic GATA1 mutations in DS newborns assessed by targeted sequencing. Our epigenome-wide association study (EWAS) of DS identifies 652 significant CpGs and 1052 differentially methylated regions (DMRs) associated with DS, including significant hypermethylation at promoter regions of RUNX1 and FLI1, both critical regulators of hematopoiesis. Further, we find that differential methylation at regulatory regions in newborns with DS correlates with gene expression patterns in DS fetal liver (FL) hematopoietic stem and progenitor cells (HSPC). This is the first multiethnic study of its kind and the largest epigenome-wide analysis of DS patients to-date, revealing insights into the etiology of DS-related phenotypes.

\section{Results}

High-quality genome-wide DNA methylation data were obtained for 196 DS and 439 non-DS newborns using Illumina Infinium MethylationEPIC Beadchip genome-wide arrays, including 651,772 CpGs on autosomes in our analyses, with an average $99.9 \% \mathrm{CpGs}$ with a detection $P$ value $<0.01$. Genome-wide copy-number analysis confirmed T21 in all DS newborns (Supplementary Fig. 1) and euploidy in all but one non-DS individual, who was excluded from subsequent analyses. Study characteristics of the 635 newborns $(N=357$ Latinos, 178 non-Latino whites, 55 Asians, 34 non-Latino blacks, and 11 other) are presented in Table 1. DS newborns had a

\section{Table 1 Baseline characteristics of newborns with and without Down syndrome included in our epigenome-wide association study.}

\begin{tabular}{|c|c|c|c|}
\hline & $\begin{array}{l}\text { Non-DS }(N=439) \\
\text { N }(\%)\end{array}$ & $\begin{array}{l}\text { DS }(N=196) \\
N(\%)\end{array}$ & $P$ value \\
\hline \multicolumn{4}{|l|}{ Sex } \\
\hline Female & $182(41.5 \%)$ & $106(54.1 \%)$ & \multirow[t]{2}{*}{$0.0034^{a}$} \\
\hline Male & $257(58.5 \%)$ & $90(45.9 \%)$ & \\
\hline \multicolumn{4}{|l|}{ Race/ethnicity } \\
\hline Asian & $38(8.7 \%)$ & $17(8.7 \%)$ & \multirow[t]{5}{*}{$0.00044^{c}$} \\
\hline Latino & $253(57.6 \%)$ & $104(53.1 \%)$ & \\
\hline Non-Latino white & $124(28.2 \%)$ & $54(27.6 \%)$ & \\
\hline Non-Latino black & $13(3.0 \%)$ & $21(10.7 \%)$ & \\
\hline Other & $11(2.5 \%)$ & $0(0 \%)$ & \\
\hline \multicolumn{4}{|c|}{ Blood collection age (days) } \\
\hline Mean (SD) & $1.33( \pm 0.70)$ & $2.49( \pm 2.04)$ & \multirow[t]{3}{*}{$<0.0001^{b}$} \\
\hline Median (range) & $1.13(0-5.25)$ & $1.75(0-15.3)$ & \\
\hline Missing & $3(0.7 \%)$ & $5(2.6 \%)$ & \\
\hline \multicolumn{4}{|c|}{ Gestational age (weeks) } \\
\hline Mean (SD) & $39.2( \pm 2.0)$ & $38.2( \pm 2.2)$ & \multirow[t]{2}{*}{$0.041^{b}$} \\
\hline Median (range) & $39.4(26.4-44.7)$ & $38.3(26.4-44.7)$ & \\
\hline $\begin{array}{l}\text { Preterm } \\
(<37 \text { weeks })\end{array}$ & $44(10.6 \%)$ & $39(22.0 \%)$ & \multirow[t]{2}{*}{$0.0004^{a}$} \\
\hline Missing & $23(5.2 \%)$ & $19(9.7 \%)$ & \\
\hline \multicolumn{4}{|l|}{ Birth weight $(\mathrm{kg})$} \\
\hline Mean (SD) & $3.39( \pm 0.55)$ & $3.01( \pm 0.73)$ & \multirow[t]{2}{*}{$0.001^{b}$} \\
\hline Median (range) & $3.41(1.04-5.05)$ & $3.01(0.96-8.65)$ & \\
\hline $\begin{array}{l}\text { Small-for- } \\
\text { gestational age }\end{array}$ & $24(6.1 \%)$ & $33(19.3 \%)$ & $<0.0001^{a}$ \\
\hline Missing & $0(0 \%)$ & $6(3.1 \%)$ & \\
\hline \multicolumn{4}{|l|}{ Birth year } \\
\hline Median (range) & $2004(2000-2008)$ & 1998 (1996-1999) & $<0.0001^{d}$ \\
\hline Missing & $3(0.7 \%)$ & $2(1.0 \%)$ & \\
\hline \multicolumn{4}{|c|}{$\begin{array}{l}\mathrm{kg} \text { kilogram, SD standard deviation, DS Down syndrome. } \\
\text { a } \mathrm{P} \text { values calculated by two-sided Fisher's exact test. } \\
\mathrm{b} P \text { values calculated by linear regression with the birth-related variable as the dependent } \\
\text { variable, DS status as the independent variable, and adjusting for the remaining birth-related } \\
\text { variables, sex, plate, and race/ethnicity. } \\
\text { cSmall-for-gestational age calculated according to the sex- and gestational age-based } \\
\text { intrauterine growth curves previously developed using US data }{ }^{85} \text {. Note we were not able to } \\
\text { calculate this for newborns born }>42 \text { weeks due to limitations of the reference data. } \\
\text { d } P \text { value calculated by the two-sided Wilcoxon rank-sum test. }\end{array}$} \\
\hline
\end{tabular}

slightly lower mean gestational age at birth $(P=0.04)$ and birth weight $(P=0.001)$ than non-DS newborns, and a higher frequency of DS newborns were preterm $(P=0.0004)$ and/or small-forgestational age $(P<0.0001)$ than non-DS newborns (Table 1$)$. Age at sampling was higher in DS newborns, the majority being sampled on day 3 of life compared to day 2 for non-DS neonates $(P<0.0001)$.

DNA methylation-based clustering separates DS and non-DS newborns. Visualization of principal components analysis (PCA) and $t$-distributed stochastic neighbor embedding ( $t$-SNE) plots generated from genome-wide DNA methylation data, excluding CpG probes on sex chromosomes and Hsa21 and CpGs overlapping single-nucleotide polymorphisms (SNPs) with minor allele frequency $(\mathrm{MAF})>0.05$, revealed clear separation of DS and non-DS newborns (Fig. 1). Intriguingly, a subset of 34 DS newborns departed from the DS cluster in the t-SNE plot, and the first PC (explaining $33.2 \%$ of overall variance) also stratified these DS newborns from the remainder. Unsupervised hierarchical clustering of the top 2000 most variable CpGs genome-wide (excluding chromosomes 21, $\mathrm{X}$, and $\mathrm{Y}$ ) resulted in similar grouping of subjects, with the first branch split separating DS from non-DS newborns, and the second split separating the same subset of 34 DS newborns among DS (Fig. 2a). Differences in blood cell proportions inferred from genome-wide DNA methylation data were seen between the three groups, as described in detail below. Two DS newborns clustered with non-DS newborns (Figs. 1 and 2) and visual inspection of copy-number 

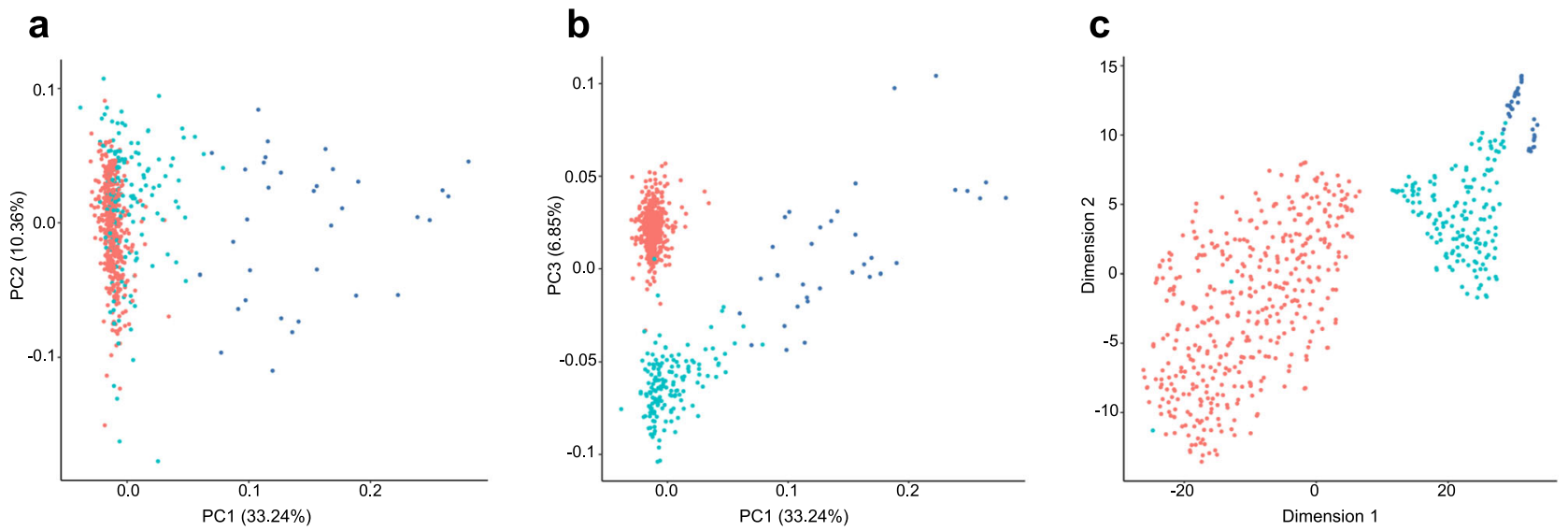

$$
\text { Non-DS DS High nRBC cluster }
$$

Fig. 1 Principal components analysis (PCA) and t-distributed stochastic neighbor embedding (t-SNE) plots of blood-based DNA methylation data in Down syndrome (DS) and non-DS newborns. PCA and t-SNE plots were generated in R, using "prcomp" and "Rtsne" functions, respectively, using genome-wide DNA methylation data from Illumina Infinium EPICmethylation Beadchip arrays in 196 DS (teal/blue) and 439 non-DS (red) newborns, excluding CpG probes on chromosomes $X, Y$, and 21. The first three principal components (PC1, PC2, and PC3) explained 33.2\%, 10.4\%, and 6.9\% of the variance, respectively. a Per-sample data for PC1 plotted against PC2. b PC1 versus PC3. DS newborns with high PC1 values also had high proportions of nucleated red blood cells (nRBCs) in deconvolution analyses; this cluster of 34 DS newborns is highlighted by the blue-colored circles in both plots. PC3 appears to be related to trisomy 21 status. c First and second t-SNE dimensions for DS (teal/blue) and non-DS (red) newborns. The cluster of 34 DS newborns with high nRBC proportions is highlighted by the blue-colored circles. Two DS newborns clustered with non-DS newborns in the PCA and t-SNE plots, and these were subsequently found to be likely mosaic for trisomy 21 (see Supplementary Fig. 2).

plots revealed that both were likely mosaic for T21 (Supplementary Fig. 2).

Targeted sequencing confirms high frequency of acquired GATA1 variants in DS neonates. We detected 34 somatic GATA1 mutations in 30 out of $184(16.3 \%)$ DS newborns assessed by targeted sequencing (Supplementary Data 1 ). The 34 mutations displayed a wide range of variant allele frequencies (VAF: $0.96-96.1 \%)$. The mean VAF of predicted functional mutations (26.3\%) was significantly higher than that of nonfunctional (noncoding/synonymous) somatic GATA1 variants $(\mathrm{VAF}=1.7 \%$, $P=0.0034$ ) (Supplementary Fig. 3). There was no significant association between the presence/absence or VAF of GATA1 mutations and sex, race/ethnicity, birth weight, gestational age, or age at blood collection (Supplementary Table 1). In the hierarchical clustering, DS newborns with GATA1 mutations with higher VAFs clustered together (Fig. 2b).

Deconvolution of blood cell proportions. Next, we used reference-based cell-type deconvolution to address whether differences in DNA methylation between DS and non-DS neonates might reflect, or be confounded by, differences in the peripheral blood cellular composition. This confirmed several of the previously reported differences in neonatal blood cell proportions in DS compared with non-DS fetuses and newborns ${ }^{8,20}$ (Fig. 2a and Supplementary Fig. 4a), with similar patterns in Latino and nonLatino white newborns (Supplementary Fig. 5), including higher proportions of erythroblasts (nucleated red blood cells (nRBCs)) $\left(P=4.45 \times 10^{-65}\right)$ and lower proportions of $\mathrm{B}$ lymphocytes $\left(P=3.48 \times 10^{-28}\right)$ and $\mathrm{T}$ lymphocytes $\left(\mathrm{CD} 4^{+} \mathrm{T}\right.$ lymphocytes) $\left(P=2.26 \times 10^{-53}\right) \quad$ (Supplementary Fig. 4a and Supplementary Table 2$)^{8,20}$. As the most dramatic difference was a subpopulation of DS newborns $(N=34,17.3 \%)$ with a high proportion of erythroblasts $(>25 \%)$, which clustered separately in Figs. 1 and 2, we next considered whether this identified the neonates with GATA1 mutations. However, although we found a higher frequency of GATA1 mutations in the newborns with increased erythroblasts compared to those with normal erythroblasts $(12 / 33,36.4 \%$ versus $18 / 151,11.9 \%, P=0.0015)$, the separate clustering of these cases is not primarily due to their GATA1 mutation status (Supplementary Figs. 4b, 6 and Supplementary Table 2). Interestingly, deconvolution analysis suggested lower proportions of monocytes $(P=$ $\left.3.75 \times 10^{-23}\right)$ and granulocytes $\left(P=8.10 \times 10^{-32}\right)$ in DS neonates. As previous studies show increased monocytes and granulocytes in DS newborns, this likely reflects the limitations of deconvolution analysis where atypical cells are present, such as blast cells and dysplastic cells, which are common in DS neonates ${ }^{8}$, and lack a suitable reference "methylome" library. Taken together, the differences in peripheral blood cell composition support the use of robust adjustment for cell-type heterogeneity in our EWAS of DS. Rather than adjusting for the blood cell proportions estimated from our reference-based deconvolution described above, we opted to include components calculated using the reference-free, sparse PCA algorithm ReFACTor (see "Methods") as covariates in our EWAS models.

Epigenome-wide significant CpGs associated with DS. To investigate the biological significance of the epigenome-wide changes in DS neonatal blood cells, we next assessed differential methylation of CpGs on autosomal chromosomes, including Hsa21, adjusted for variation in cell-type proportions, sex, and ancestry-informative PCs. A total of 652 DS-associated CpGs were detected following Bonferroni correction $\left(P<7.67 \times 10^{-8}\right.$, Fig. 3a and Supplementary Data 2), with 319/652 (48.9\%) CpGs hypermethylated and 333/652 (51.1\%) hypomethylated in DS compared with non-DS newborns. The pattern of DNA methylation was distinctly different for CpGs on Hsa21; the majority of significantly differentially methylated CpGs $(64 / 79,81.0 \%)$ were hypomethylated, whereas on other chromosomes, the proportions of hypomethylated $\mathrm{CpGs}$ were on average much lower (median: $43.5 \%$, range: $16.0-80.0 \%$ ). In addition, when considering all $\mathrm{CpGs}$ included in the EWAS, a significantly higher proportion of probes on Hsa21 were hypomethylated (4425/7351, $60.2 \%$ ) compared with probes on all other autosomes combined 

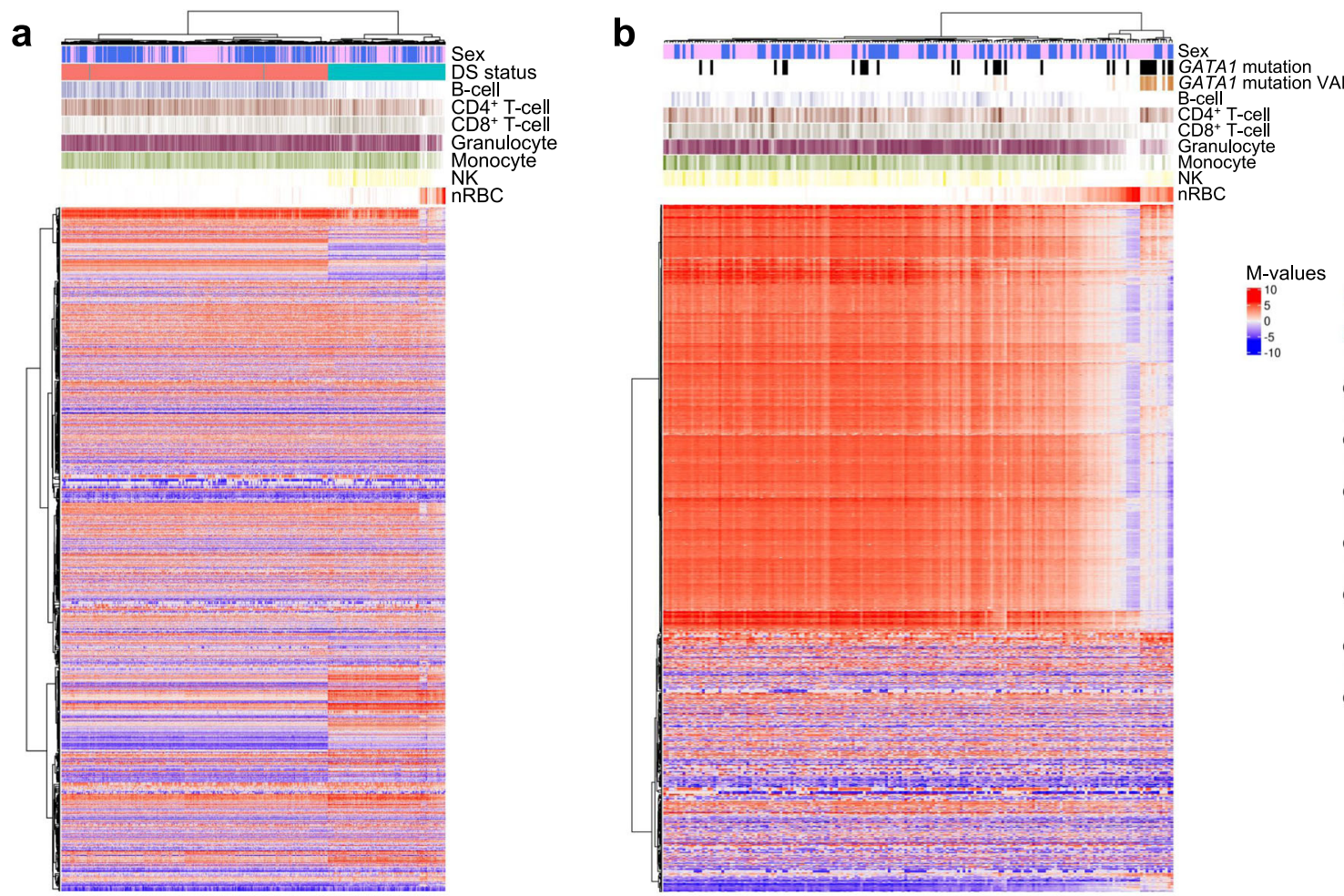

Fig. 2 Heatmap and unsupervised hierarchical clustering of the top 2000 most variable CpGs genome-wide in Down syndrome (DS) and non-DS newborns, and by GATA1 mutation status. These plots were generated using genome-wide DNA methylation data excluding chromosomes $\mathrm{X}, \mathrm{Y}$, and 21 , and with $\beta$ values converted to $M$ values, using the "ComplexHeatmap" R package. Only the top $2000 \mathrm{CpGs}$ with the greatest mean absolute deviation are displayed. a Hierarchical clustering and heatmap of 196 DS and 439 non-DS newborns, in relation to subject sex and deconvoluted blood cell proportions of $\mathrm{B}$ cells, CD4 ${ }^{+} \mathrm{T}$ cells, CD8 ${ }^{+} \mathrm{T}$ cells, granulocytes, monocytes, natural killer (NK) cells, and nucleated red blood cells (nRBCs). The first separation in hierarchical clustering represented DS (teal) versus non-DS (red) newborns. Two DS newborns clustered with the non-DS newborns, and were the same as those that clustered similarly in the t-SNE plot in Fig. 1. The DS newborns clustered into two main subgroups, those with high nRBC proportions and those with low nRBC proportions. b Hierarchical clustering and heatmap of 184 DS newborns, including 30 found to harbor somatic GATA1 mutations via targeted sequencing, and 154 found to be GATA1 mutation wildtype. DS newborns with GATA1 mutations are represented by black bars, and the variant allele frequency (VAF) range of mutations shown in the row below. The first split appeared to separate DS newborns with high nRBC proportions from those with low nRBC proportions, whereas the second split was largely driven by subjects with GATA1 mutations with high VAF. DS newborns with GATA1 mutations with high VAF clustered together and tended to have high nRBC proportions; however, a proportion of GATA1 wild-type newborns were also found to have high nRBC proportions, and these also clustered separately.

(356,222/644,421, 55.3\%, $P<0.0001$, Chi-squared test), which was particularly the case in shores and shelves but not in CpG islands themselves (Supplementary Table 3).

Remarkably, 13 of 15 hypermethylated and epigenome-wide significant Hsa21 CpGs overlapped RUNX1, at the proximal P2 promoter (Supplementary Fig. 7). RUNX1 was also identified when we considered the epigenome-wide significant $\mathrm{CpGs}$ that overlapped known genes $(N=357)$; of the top 20 significant CpGs, overlapping 11 unique genes, the three with the largest methylation differences (beta coefficients $>0.38$ ) were all located in RUNX1 (Table 2 and Supplementary Data 2). The other ten genes included the megakaryocytic gene FLI, SH3D21, and KIAA0087 (each with two CpGs), and DST, VSIG2, KLF16, OLFML1, SETD3, CELF3, and NOL10 (one CpG). Of the four intergenic CpGs, two overlap a putative enhancer of HES1 $(\text { Table } 2)^{21}$. We repeated linear regression analyses for the top significant CpGs in RUNX1 (cg12477880) and FLI1 (cg17239923), genes that are known regulators of hematopoiesis, adjusting for deconvoluted blood cell proportions instead of ReFACTor components (see "Methods"), and the associations with DS remained highly significant (both $P<2.0 \times 10^{-16}$ ).

Neither removal of DS newborns with GATA1 mutations $(N=$ $30)$ nor those with high erythroblasts $(N=34)$ affected EWAS results substantially, and ethnicity-stratified analyses showed similar results, with 622/652 (95.4\%) epigenome-wide significant CpGs showing the same direction of effect in both Latinos and non-Latino whites (Supplementary Data 2). In addition, in a subset of newborns with available birth weight and gestational age information (176 DS and 416 non-DS), we repeated the EWAS adjusting for these birth variables, but again, the results were not substantially altered (Supplementary Data 2). In a sex-stratified EWAS, no chromosome X CpGs were epigenome-wide significant in females, and 2 significant CpGs in males did not replicate in females (Supplementary Data 2).

We also determined the overlap of DS-associated CpGs with genomic locations and functional elements. Hypomethylated CpGs were significantly underrepresented at gene promoters and $\mathrm{CpG}$ islands (Supplementary Fig. 8 and Supplementary Data 3). Hypermethylated CpGs were significantly enriched at binding sites for NFE2, MAFF, MAFK, and BACH1, TFs that form components of a key erythro-megakaryocyte regulatory network (Supplementary Data 3). Hypermethylated CpGs were also significantly enriched at DNase I hypersensitive sites (DHS) $\left(P=1.67 \times 10^{-16}\right)$, at H3K4me1- and H3K4me3-binding sites in hematopoietic stem cells (HSCs, both $P<5.73 \times 10^{-10}$ ), and at enhancer loci in HSCs $\left(P<3.27 \times 10^{-6}\right)$, whereas hypomethylated $\mathrm{CpGs}$ were significantly enriched at H3K36me3 sites $\left(P=9.92 \times 10^{-11}\right)$ (Supplementary Data 3), altogether indicating repression of gene expression. 
a

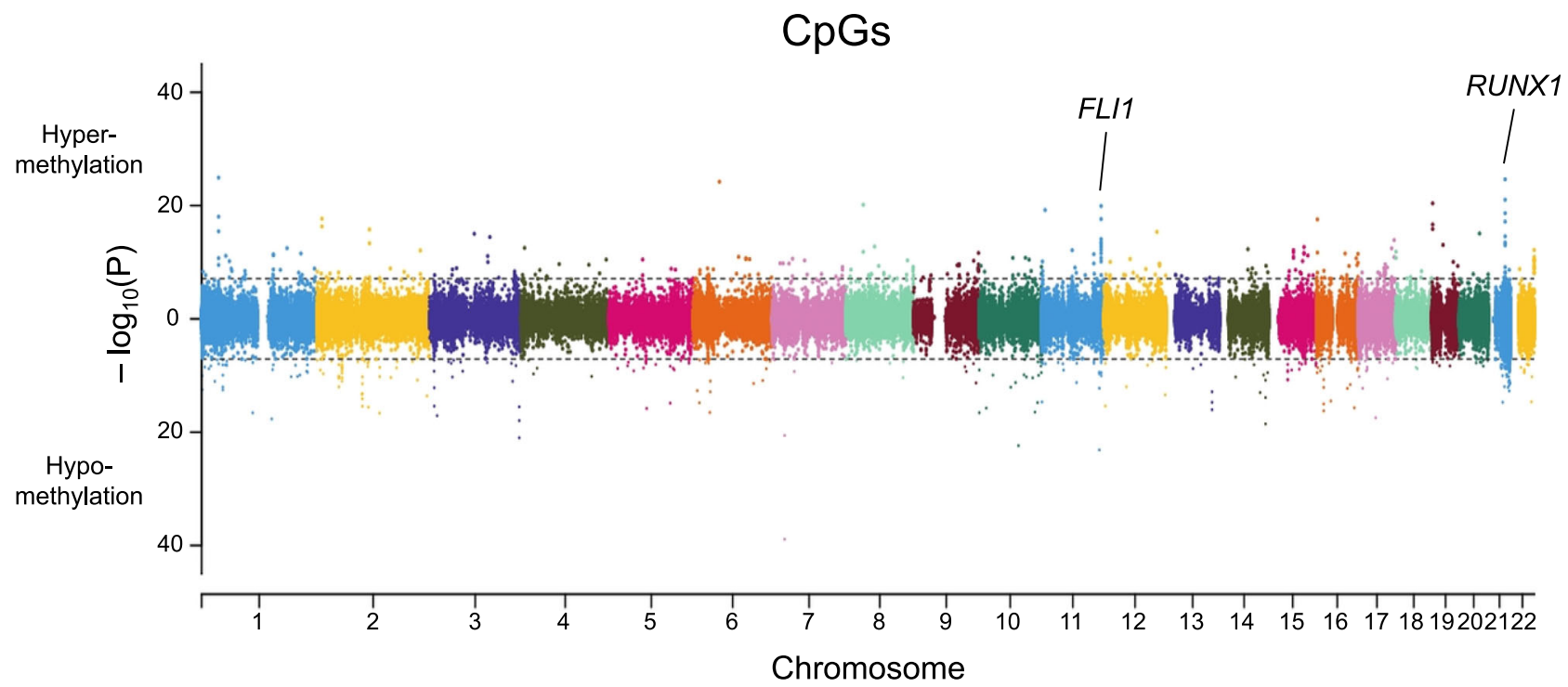

b

\section{DMRs}

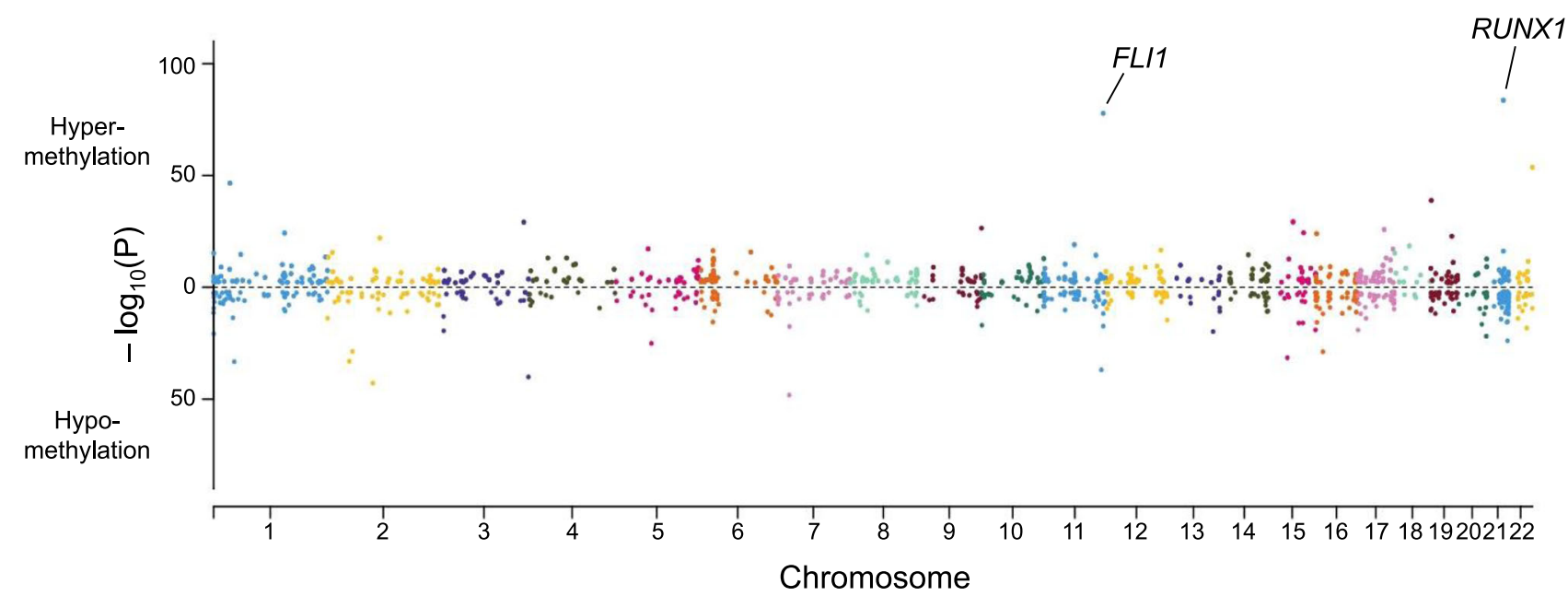

Fig. 3 Bidirectional Manhattan plots displaying Down syndrome (DS)-associated CpGs and differentially methylated regions (DMRs) genome-wide. a Manhattan plot presenting the $-\log _{10}(P)$ values of autosomal $\mathrm{CpGs}$ derived from the multiethnic epigenome-wide association study (EWAS) of DS. $P$ values were calculated using linear regression testing the association of each $\mathrm{CpG}$ beta-value with DS, adjusting for sex, plate, the first ten ReFACToR principal components (PCs), and the first ten EPISTRUCTURE PCs. Points above zero correspond to CpGs that were hypermethylated in DS ( $N=196)$ versus non-DS $(N=439)$ newborns, whereas points below zero correspond to hypomethylated CpGs in DS. The genomic inflation factor $(\lambda)$ was 1.47 . Dotted lines correspond to the threshold for epigenome-wide significance $\left(P=7.67 \times 10^{-8}\right)$ after Bonferroni correction for multiple testing. The strong association peaks at FLI1 on chromosome 11 and RUNX1 on chromosome 21 are highlighted. b Manhattan plot showing DMRs identified in the overall multiethnic analysis using DMRcate and comb-p. The $-\log _{10}(P)$ values were derived from the Šidák-corrected $P$ values from the more stringent method, comb-p, with the DS EWAS $P$ values at each $\mathrm{CpG}$ as input. Points above zero correspond to DMRs with a mean $\Delta \beta$-value above 0 (hypermethylated in DS), whereas points below zero correspond to hypomethylated DMRs in DS. The top two significant DMRs in RUNX1 and FLI1 are highlighted. We identified DMRs in several additional genes that regulate hematopoiesis and/or are known drivers of leukemogenesis, including at Hsa21 genes DYRK1A (DMR N =3), ERG $(N=2)$, and $E T S 2(N=1)$, and at non-Hsa21 genes ETV6 $(N=3), B C R$ and KIT (both $N=2$ ), and GATA2, TET1, TET2, FLT3, DNMT3A, KAT6B, KMT2C, PBX1, and RARA (all N=1) (Supplementary Data 7).

Pathway analysis of genes overlapped by epigenome-wide significant $\mathrm{CpGs}$ revealed significant enrichment for 62 Gene Ontology (GO) terms, the majority related to hematopoiesis and immune function, and 12 Kyoto Encyclopedia of Genes and Genomes (KEGG) pathways, with hematopoietic cell lineage displaying the strongest enrichment in addition to several immune-related pathways (Supplementary Data 4).
We next assessed whether previously reported DS-associated CpGs were replicated. In two previous studies, from Bacalini et al. and Henneman et al. ${ }^{16,17}$, there were 111 DS-associated CpGs with concordant directions of effect. Of these, 97 were present on the EPIC array and passed quality control (QC) filtering, and 74/97 were associated with DS at $P<0.05$ and all with the same direction of effect (Supplementary Data 5). 
Table 2 Top differentially methylated CpG probes associated with Down syndrome.

\begin{tabular}{|c|c|c|c|c|c|c|}
\hline 1 & 36786777 & SH3D21 & cg02993069 & 0.198 & $1.14 \times 10^{-25}$ & Yes \\
\hline 6 & 56607099 & DST & cg08882472 & 0.157 & $6.14 \times 10^{-25}$ & \\
\hline 11 & 124621829 & VSIG2 & cg24942416 & -0.175 & $7.33 \times 10^{-24}$ & \\
\hline 10 & 85363826 & Intergenic & cg07841633 & -0.329 & $4.21 \times 10^{-23}$ & \\
\hline 21 & 36259383 & $R \cup N X 1$ & cg00994804 & 0.388 & $9.36 \times 10^{-22}$ & Yes \\
\hline 19 & 1851882 & KLF16 & cg13382072 & 0.180 & $4.07 \times 10^{-21}$ & Yes \\
\hline 8 & 37575051 & Intergenic & cg24020235 & 0.188 & $7.30 \times 10^{-21}$ & \\
\hline 11 & 128556611 & FLI1 & cg17239923 & 0.232 & $1.21 \times 10^{-20}$ & \\
\hline 11 & 7519636 & OLFML1 & cg19030331 & 0.165 & $6.05 \times 10^{-20}$ & \\
\hline 21 & 36258497 & RUNX1 & cg03142697 & 0.396 & $2.15 \times 10^{-19}$ & \\
\hline 14 & 99880641 & SETD3 & cg24999883 & -0.067 & $2.85 \times 10^{-19}$ & \\
\hline 11 & 128556341 & FLI1 & cg19765472 & 0.207 & $2.42 \times 10^{-18}$ & \\
\hline
\end{tabular}

Finally, an EWAS of GATA1 mutations (presence/absence) in DS newborns revealed 13 epigenome-wide significant CpGs (Supplementary Data 6), 12 of which were hypomethylated in GATA1 mutation-positive DS newborns, although all had beta coefficients $<0.10$. No sex chromosome CpGs were associated with GATA1 mutations in females or males.

DMRs associated with DS. We identified 1052 DMRs associated with DS across the genome (Fig. 3b and Supplementary Data 7), following adjustment for variation in cell-type proportions, sex, and ancestry-informative PCs. DMRs were identified on all chromosomes, with a particularly high proportion $(11.2 \%)$ on Hsa21 (Supplementary Fig. 9). The 1052 DMRs overlapped 943 unique genes, and 291/1052 (27.7\%) overlapped promoter regions. The top 20 most significant DMRs (Table 3) overlapped 17 genes, with the top two again including the key hematopoietic TF genes RUNX1 $\left(P=2.30 \times 10^{-84}\right)$ and FLI1 $\left(P=1.65 \times 10^{-78}\right)$. The FLI1 DMR overlapped the promoter of transcript variant 4 , which is largely expressed in cord blood megakaryocytes relative to other blood cell types, a pattern not found for other FLI1 transcript variants in gene expression data in BLUEPRINT (Supplementary Fig. 10) ${ }^{22}$. The top 20 DMRs remained significant following removal of DS newborns with high erythroblasts $(N=34)$ or removal of GATA1 mutation-positive individuals $(N=30)$, and in ethnicity-stratified analyses with the exception of CCDC17, ANAPC2, and MIR1224, which were not detected in non-Latino whites.

Of six DMRs with at least ten CpG probes and with mean $\Delta \beta$-value $>0.10$ (Table 3 ), two overlapped regulatory regions in RUNX1 (Fig. 4a) and FLI1 (Fig. 5a), and the remaining four DMRs overlapped promoter regions of genes involved in brain development (CPT1B, CMYA5, and PRDM8) and the immune system (ASB3) (Supplementary Fig. 11 and Table 3). Assessment of genome-wide association study (GWAS) Catalog SNPs revealed that 8 of the top 20 DMRs are nearby SNPs associated with hematological traits, with 7 DMRs nearby SNPs associated with brain-related traits (Table 3 and Supplementary Data 8).

We next assessed overlap between our DS-associated DMRs with those reported in the previous largest EWAS of $\mathrm{DS}^{16}$. Of the
66 DMRs previously identified with a large $\beta$-value difference $(>0.15)$, we also detected 37 as DMRs, all of which had concordant $\Delta \beta$-value directions (Supplementary Data 5).

In addition, we identified 59 DMRs associated with GATA1 mutations in DS neonates (Supplementary Data 6); the most significant region encompassed the noncoding RNA VTRNA2-1 $\left(P=1.71 \times 10^{-20}\right)$, with reduced DNA methylation (mean $\Delta \beta$ value $=-0.115)$ in GATA1 mutation-positive DS newborns versus wild-type DS newborns. DNA methylation in DS newborns at the top two DS-associated DMRs, in RUNX1 and FLI1, was not driven by the presence of GATA1 mutations; in fact, at both DMRs, which were significantly hypermethylated in DS newborns, the mean methylation levels were slightly lower in GATA1 mutation-positive than in wild-type DS newborns, albeit still considerably higher than in non-DS newborns (Supplementary Figs. 12 and 13).

Gene expression changes in DS versus non-DS FL CD34+ cells. FL is the main site of hematopoiesis until birth and neonatal blood is likely derived from FL HSPC. To ascertain whether differences in genome-wide DNA methylation found in neonatal T21 blood cells correlate with differences in gene expression, we analyzed RNAsequencing data from DS $(N=3)$ and non-DS $(N=3)$ FL HSPC. We found 587 significantly differentially expressed genes between DS and non-DS FL CD34+ cells $($ FDR $<0.1)$, of which 294 genes were upregulated and 293 downregulated in DS (Fig. 6a and Supplementary Data 9). DS-associated DMRs identified at promoter or enhancer regions in neonatal blood $(N=729$, Supplementary Data 7) overlapped 491 genes that demonstrated any change in expression in DS FL cells compared with non-DS cells; hypermethylation at these DMRs correlated with decreased gene expression in DS FL CD34+ cells, whereas hypomethylation correlated with increased gene expression $(P<0.0001$, two-tailed Fisher's exact test) (Fig. $6 \mathrm{~b}$ and Supplementary Data 7 ), a relationship that remained when limiting to the significantly differentially expressed genes $(P=0.0002)$ or after excluding Hsa21 genes $(P<0.0001)$. Conversely, no relationship between hyper-/hypomethylation and gene expression was found for the 323 DMRs outside of promoters/enhancers (Supplementary Fig. 14). 


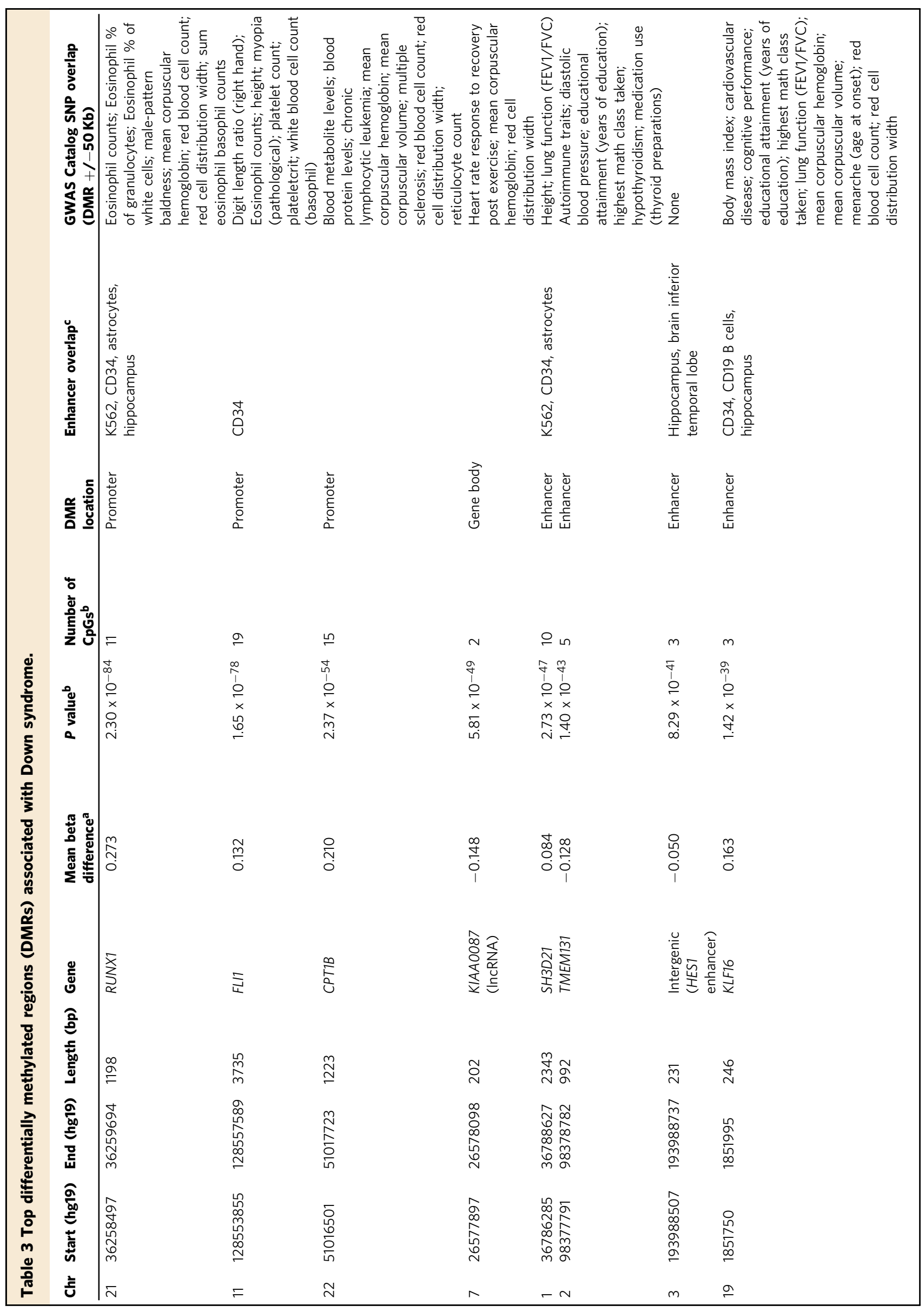




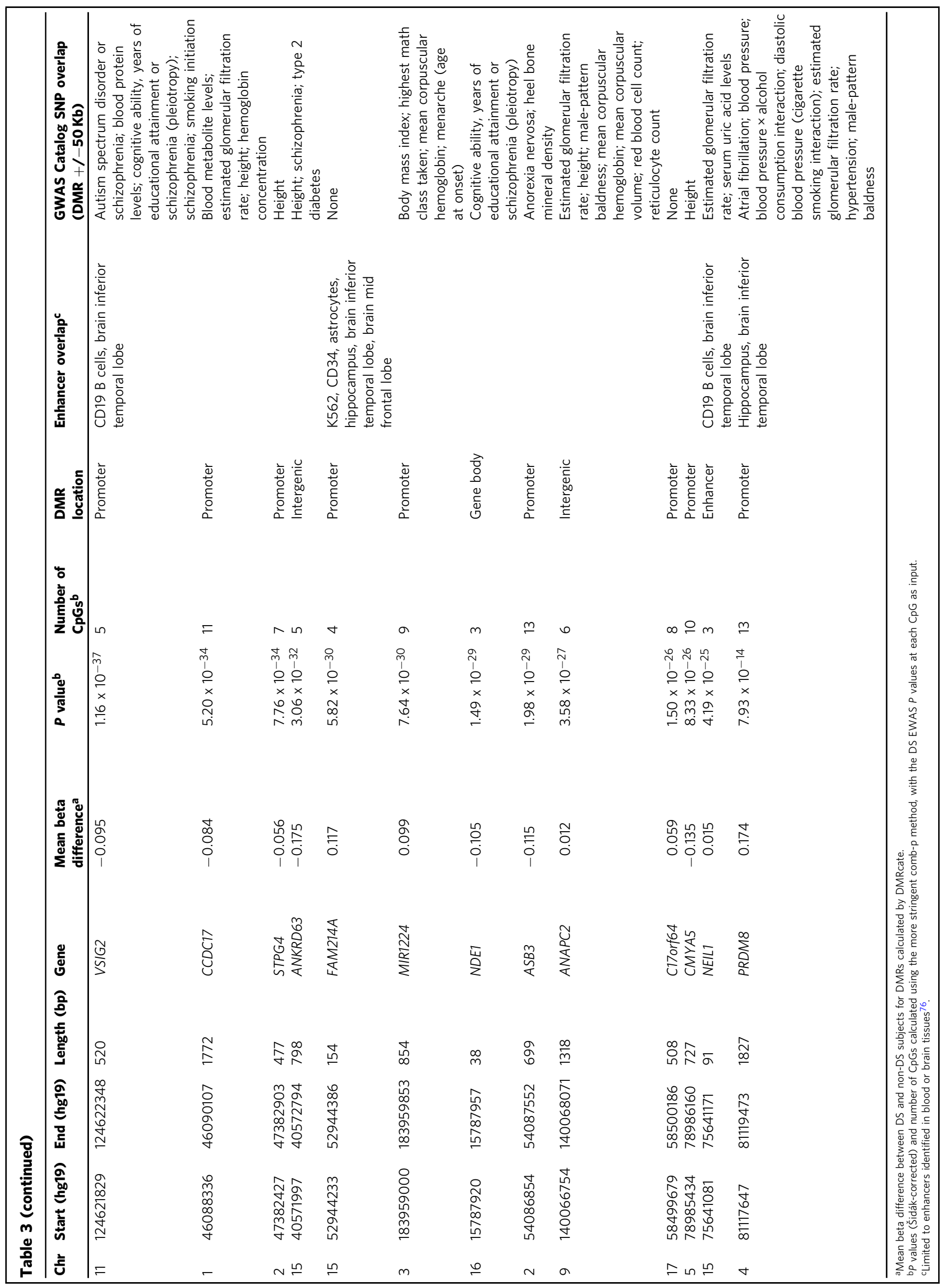




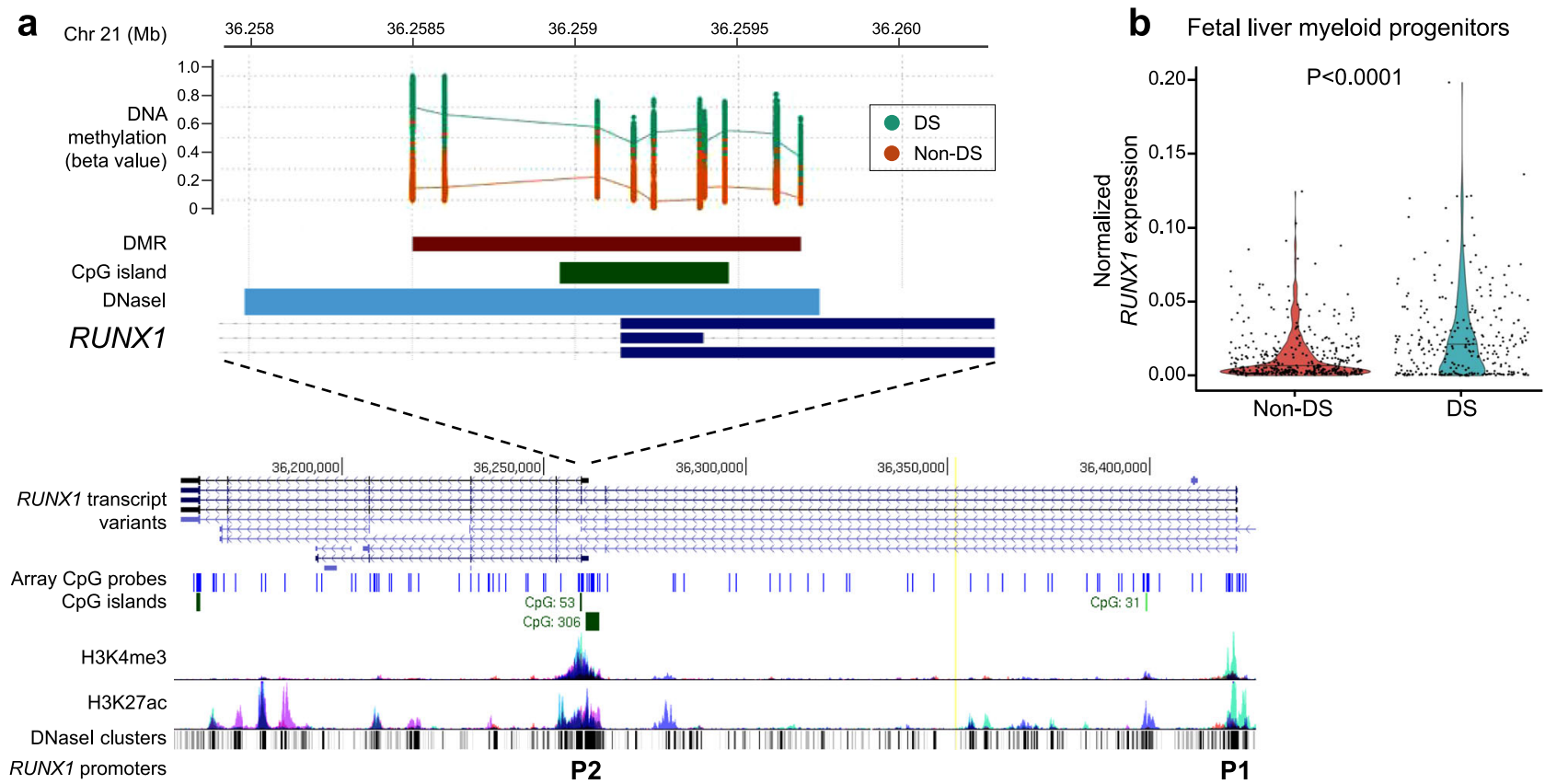

Fig. 4 Down syndrome (DS)-associated differentially methylated region (DMR) overlapping RUNX1 regulatory region. a DS-associated DMR (Šidákcorrected $P$ value $=2.30 \times 10^{-84}$ from comb-p), which included $11 \mathrm{CpGs}$, had the greatest mean $\Delta \beta$-value $(+0.273)$ between DS (teal, $\left.N=196\right)$ and nonDS (red, $N=439$ ) newborns, and overlapped a large regulatory region that encompasses the RUNX1 proximal P2 promoter and the first exon of the P2 isoform, or exon 4 of the P1 isoform. The position of the DMR (brown horizontal bar) is shown relative to the RUNX1 gene in the UCSC Genome Browser (https://genome.ucsc.edu/), along with tracks for chromatin accessibility (DNase I clusters, darkness corresponds to signal strength) and histone modifications (H3K4me3, H3K27ac), CpG islands (green), and a custom track displaying positions of the array CpG probes (blue). High coverage of CpG probes is shown both at the proximal P2 and distal P1 promoters of RUNX1. b Violin plot showing normalized RUNX1 expression derived from single-cell qRT-PCR on index-sorted FL myeloid progenitors with megakaryocyte-erythroid potential (Lin-CD34+CD38+CD45RA-) from non-DS $(N=3)$ and DS $(N=3)$ subjects. RUNX1 expression was significantly increased in DS myeloid progenitor cells $(N=292)$ compared with non-DS cells $(N=412)$ $\left(P=3.81 \times 10^{-5}\right.$, two-sided Wilcoxon rank-sum test $)$. Horizontal lines represent the median.

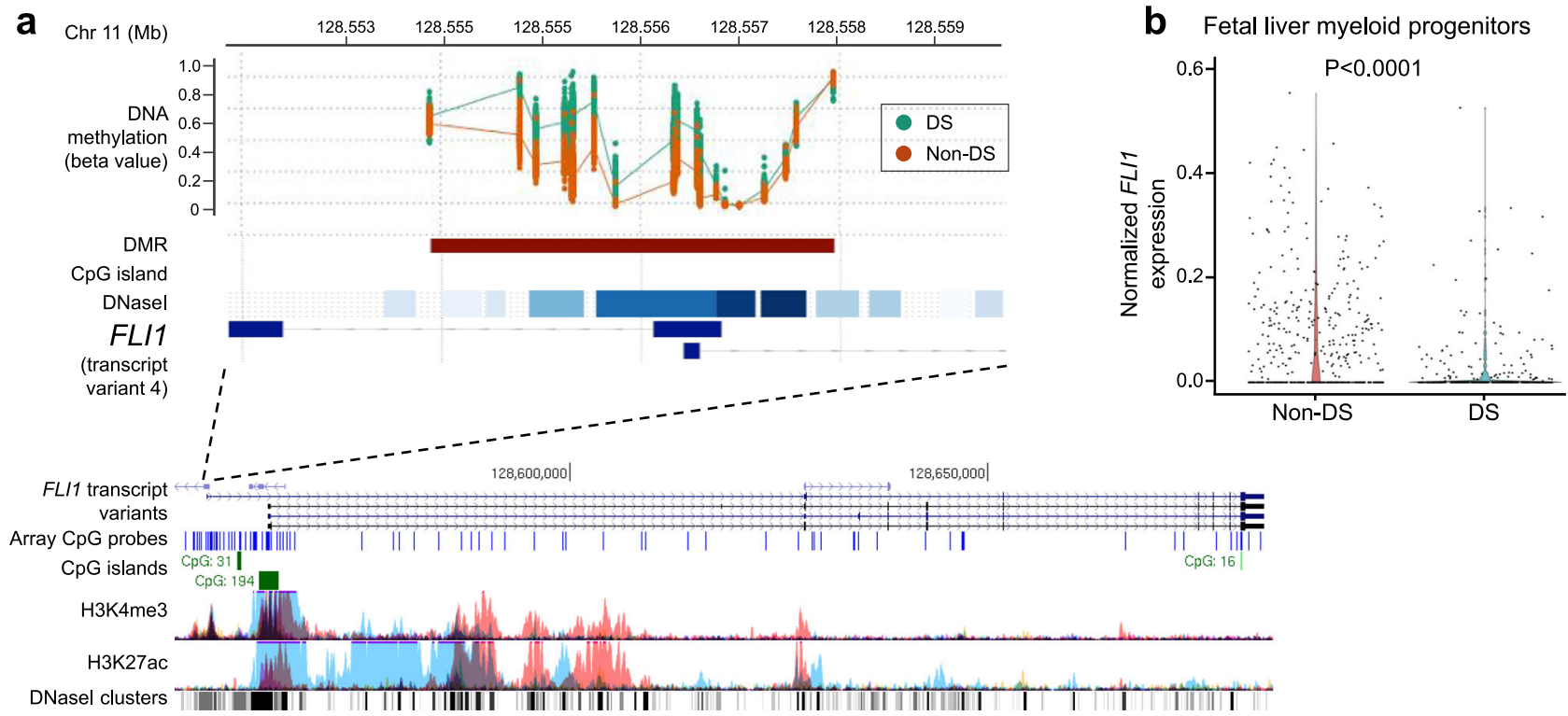

Fig. 5 Down syndrome (DS)-associated differentially methylated region (DMR) overlapping FLI1 promoter region. a DS-associated DMR (Šidákcorrected $P=1.65 \times 10^{-78}$ from comb-p), which included $19 \mathrm{CpGs}$, had a mean $\Delta \beta$-value $=+0.132$ between DS (teal, $\left.N=196\right)$ and non-DS (red, $N=439$ ) newborns, and overlapped a promoter of FLI7 transcript variant 4. The position of the DMR (brown horizontal bar) is shown relative to the FLI1 gene in the UCSC Genome Browser (https://genome.ucsc.edu/), along with tracks for chromatin accessibility (DNase I clusters, darkness corresponds to signal strength) and histone modifications (H3K4me3, H3K27ac), CpG islands (green), and a custom track displaying positions of the array CpG probes (blue). b Violin plot showing normalized FLI1 expression derived from single-cell qRT-PCR on index-sorted FL myeloid progenitors with megakaryocyte-erythroid potential ( $\mathrm{Lin}-\mathrm{CD} 34+\mathrm{CD} 38+\mathrm{CD} 45 \mathrm{RA}-)$ from non-DS $(N=3)$ and DS $(N=3)$ subjects. FL/1 expression was significantly reduced in DS myeloid progenitor cells $(N=292)$ compared with non-DS cells $(N=412)\left(P=2.20 \times 10^{-16}\right.$, two-sided Wilcoxon rank-sum test). Horizontal lines represent the median. 

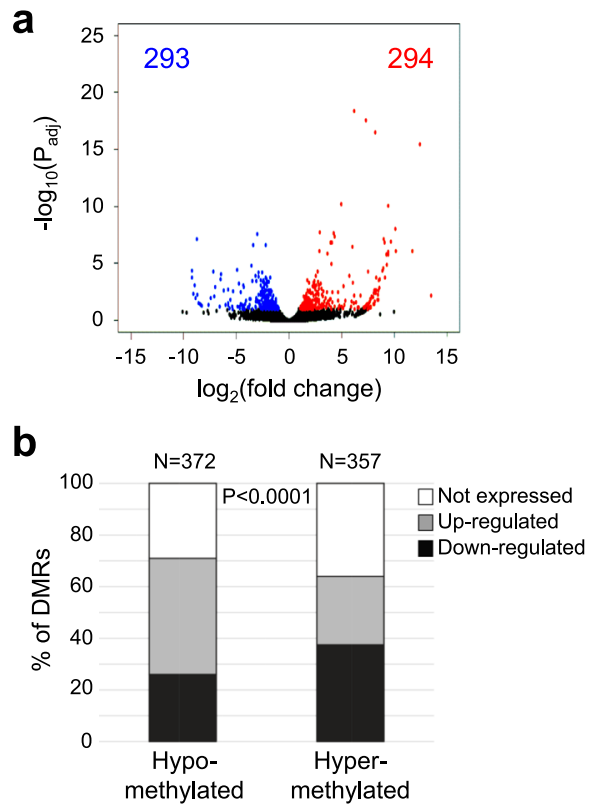

C
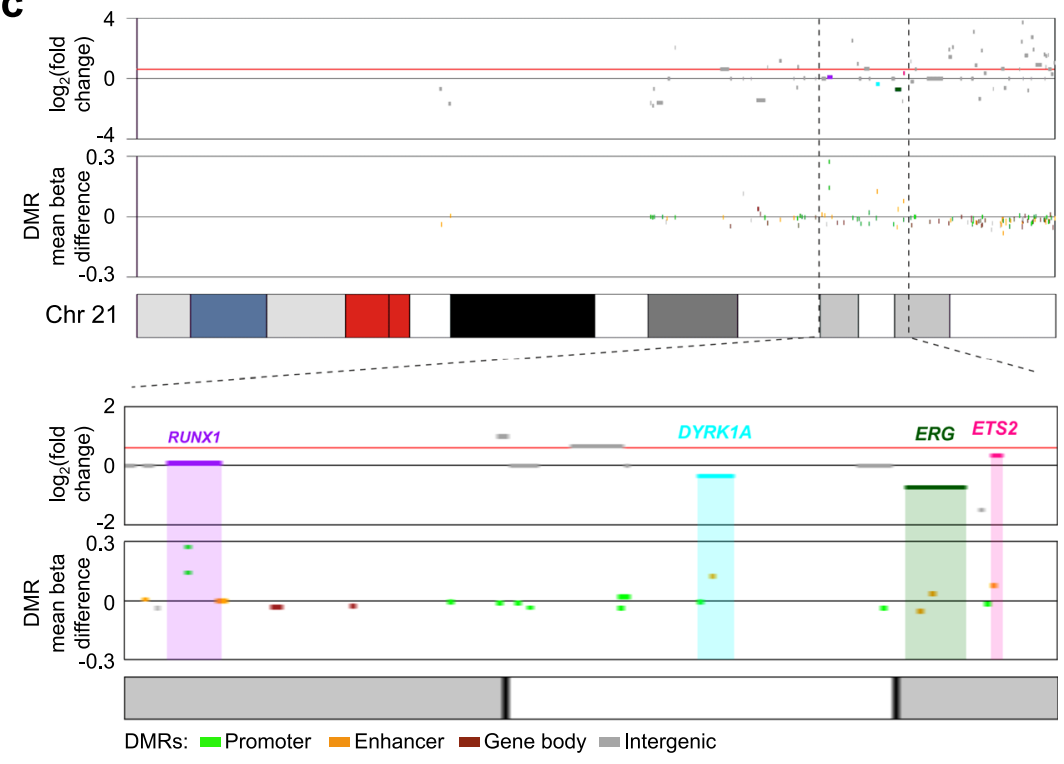

Fig. 6 Correlation between differential methylation in newborn dried bloodspots and differential gene expression in fetal liver CD34 + cells in Down syndrome (DS) and non-DS samples. a Volcano plot showing differential gene expression between DS $(N=3)$ and non-DS $(N=3)$ FL CD34+ cells, with significantly upregulated and downregulated genes (FDR-adjusted $P<0.1)$ highlighted in red $(N=294)$ and blue $(N=293)$, respectively. b Bar chart showing the number of hypomethylated $(N=372)$ and hypermethylated $(N=357)$ DS-associated differentially methylated regions (DMRs) that overlapped gene promoters/enhancers, and the direction of differential expression of the corresponding genes in DS versus non-DS FL CD34+ cells. For hypomethylated DMRs, 96 genes were downregulated and 167 genes upregulated (109 genes not expressed), whereas for hypermethylated DMRs, 134 genes were downregulated and 94 genes upregulated (129 not expressed), a difference that was highly significant in a two-sided Fisher's exact test $(P=$ $9.20 \times 10^{-7}$ ). c DS-associated DMR methylation levels and corresponding differential gene expression levels across chromosome 21 . The zoomed-in plot below highlights four genes that play an important role in hematopoiesis, RUNX1, DYRK1A, ERG, and ETS2, all of which were overlapped by hypermethylated DMRs in promoters/enhancers and also demonstrated less than the expected 1.5-fold change in expression (indicated by the red horizontal line) in DS compared to normal FL CD34+ cells.

We next took a closer look at DMRs and gene expression on Hsa21. While an extra copy of Hsa21 would predict for a 1.5-fold increased expression of the genes on this chromosome, this is often not the case suggesting that epigenetic mechanisms regulate gene expression in the context of aneuploidy. Here, as for nonHsa21 genes, we found that Hsa21 changes in gene expression in DS fetal hematopoietic cells negatively correlated with DNA methylation status at promoter/enhancer regions in DS neonatal hematopoietic cells (Fig. 6c and Supplementary Fig. 14). Several Hsa21 genes that play a key role in hematopoiesis, such as RUNX1, ERG, DYRK1A, and ETS2 showed less than the expected 1.5 -fold change when compared to normal FL, and this was accompanied by significant hypermethylation at promoters/ enhancers of these genes in neonatal blood (Supplementary Data 7 and Fig. 6c).

Finally, we analyzed the expression of RUNX1 and FLI1, the top 2 genes with DMRs and both essential regulators of megakaryopoiesis, via single-cell $\mathrm{qRT}-\mathrm{PCR}$ on index-sorted DS and non-DS FL myeloid progenitors with megakaryocyte-erythroid potential (Lin-CD34+CD38+CD45RA-). This showed markedly lower expression of FLI1 in DS FL myeloid progenitors $(P<0.0001)$, consistent with hypermethylation of the FLI1 promoter in DS neonatal blood (Fig. 5b), while RUNX1 expression was increased in DS myeloid progenitors $(P<0.0001)$ compared to normal $\mathrm{FL}$ counterparts (Fig. 4b), perhaps reflecting the significant DMR hypermethylation found at the RUNX1 P2 promoter but not the P1 promoter in DS newborns (Supplementary Fig. 7).

\section{Discussion}

We report the results from the largest, and first multiethnic, EWAS of DS in blood cell samples at birth, confirming several known loci and identifying many novel regions, including at FLI1, that were significantly differentially methylated in newborns with DS compared with newborns without DS. The 652 epigenomewide significant CpGs and 1052 DMRs demonstrate the profound epigenome-wide consequences of T21, which likely contribute toward phenotypic variation in DS. The majority of DSassociated DNA methylation changes were found on euploid (non-21) chromosomes, as previously reported ${ }^{15-17}$, supporting that T21 results in genome-wide perturbations in gene regulation ${ }^{12,23}$. Indeed, our results from RNA sequencing of fetal DS and non-DS HSPCs support the early-life, genome-wide perturbation of gene expression in hematopoietic cells that broadly correlates with differential DNA methylation patterns in DS.

The effects of T21 on the function of RUNX1, a crucial regulator of hematopoiesis particularly in early development ${ }^{24}$, appear complex. Although DS was largely associated with hypomethylation on Hsa21, we found significant hypermethylation at RUNX1, as reported previously in DS and in DS-ALL ${ }^{16-18,25}$. We note that RUNX1 hypermethylation in DS was specific to the proximal P2 promoter ${ }^{26}$, which is thought to be the dominant regulator of RUNX1 expression during embryonic development, driving formation of the hemogenic endothelium and early hematopoiesis 27,28 . Dosage of RUNX1 during these early stages is tightly controlled ${ }^{29,30}$, suggesting that RUNX1 downregulation via $\mathrm{P} 2$ promoter hypermethylation may be required for viable embryo development in DS. The distal P1 promoter becomes active once cells commit to the hematopoietic lineage and is the predominant promoter in definitive hematopoiesis ${ }^{27,28}$, consistent with the pattern of RUNX1 expression we observed in DS FL myeloid progenitors. Promoter switching from P2 to P1 
involves changes in DNA methylation at the P1 promoter but not at P2, which was found to be unmethylated across cell types ${ }^{31}$, suggesting that $\mathrm{P} 2$ hypermethylation is unique to DS.

The most significant DMR outside of Hsa21 overlapped FLI1, another important regulator of megakaryopoiesis ${ }^{32,33}$, specifically at the promoter of transcript variant 4 that is mainly expressed in megakaryocytes. FLI1 protein is a critical binding partner of both RUNX1 and GATA1 during terminal megakaryocyte maturation ${ }^{34,35}$ and all three proteins cooperate in transcriptional control of megakaryocyte differentiation ${ }^{36}$. Similar to RUNX1, germline loss of FLI1 has been associated with thrombocytopenia ${ }^{33}$, defects in megakaryopoiesis ${ }^{37}$, and familial platelet disorders ${ }^{38}$. We report that FLI1 expression is significantly reduced in DS FL myeloid progenitor cells. Our results support that T21 leads to epigenetic dysregulation of both RUNX1 and FLI1, which may contribute toward abnormal megakaryocyte development in DS FL cells ${ }^{3}$, and to the development of TAM and the concomitant risk of AMKL in DS infants. The etiology and timing of these epigenetic changes remain to be determined. Along with RUNX1, FLI1 is also a critical regulator of embryonic hematopoiesis ${ }^{24,39}$; thus, compensatory epigenetic downregulation of RUNX1 and FLI1 may be required for viable embryogenic development in DS, but potentially also results in increased risk of hematological malignancies.

Our results confirm previous studies that pinpointed RUNX1 as one of the most differentially methylated genes in blood in individuals with $\mathrm{DS}^{16,17}$. It is interesting that DS-associated hypermethylation at $R U N X 1$ has also been reported in DS brain tissue $^{18,40}$, supporting the early fetal origins of these epigenetic changes and potential pleiotropic effects on DS phenotypes. Indeed, $R U N X 1$ has been shown to play a role in proliferation and differentiation of select neural progenitor cells, including in hippocampal precursor cells ${ }^{41,42}$. The overlap of DS-associated DMRs with GWAS loci for both cognitive-related and hematological traits, such as at KLF16, further supports the possibility that epigenetic dysregulation may underlie both hematologic defects and cognitive development in DS. Remarkably, two of the most significant DMRs, overlapping promoters of $C P T 1 B$ and CMYA5, were recently associated with hippocampal volume in non-DS individuals; for both DMRs, the direction of DNA methylation changes in DS newborns was associated with smaller hippocampal volume ${ }^{43}$. Additional DS-associated DMRs overlapped $N D E 1, P R D M 8$, and the enhancer locus for HES1, genes that all play an important role in neurogenesis ${ }^{44-46}$.

Cell-type deconvolution revealed that DS newborns had relatively high proportions of erythroblasts, possibly indicative of intrauterine or perinatal hypoxia ${ }^{47}$, pulmonary hypertension ${ }^{48}$, or $\mathrm{TAM}^{49}$. Although no DS newborns in this study developed childhood leukemia ${ }^{50}$, targeted GATA1 sequencing identified that $\sim 14 \%$ harbored a likely functional somatic GATA1 mutation, consistent with the observation that the majority of DS newborns with TAM and Silent TAM will not develop AMKL ${ }^{8}$. We found significant association between GATA1 mutations and higher erythroblast proportions; however, almost two-thirds of DS newborns with high erythroblast proportions did not harbor GATA1 mutations, suggesting a greater role for pre- and perinatal hypoxic conditions in contributing to this phenotype.

Nonfunctional GATA1 variants tended to have much lower VAF than functional ones, supporting that GATA1-truncating mutations confer a growth advantage to fetal hematopoietic cells and are clonally selected during development of TAM. Moreover, the true frequency at which somatic GATA1 mutations arise in utero may be higher than detected at the current limits of detection and by sampling blood at birth. The etiology of GATA1 mutations in DS remains unknown, but is potentially related to
T21-associated upregulation of GATA1 ${ }^{3}$; increased transcription is a known cause of DNA mutagenesis ${ }^{51}$. Intriguingly, human adaptation to hypoxic conditions includes upregulation of GATA1 to drive erythropoiesis ${ }^{52}$. Thus, hypoxic intrauterine conditions in developing DS fetuses may contribute to the generation of GATA1 mutations or at least to expansion of mutant GATA1 clones $^{53}$. Our EWAS of GATA1 mutations in DS revealed a DMR overlapping VTRNA2-1, a metastable epiallele at which DNA methylation levels were previously associated with the periconceptional environment ${ }^{54}$, suggesting a potential environmental role in the development of GATA1 mutations.

An important strength of our study was the use of newborndried bloodspots (DBS), which increased our power to detect differentially methylated loci associated with DS, as epigenetic influences of environmental exposures and age-related changes as well as drift would be much reduced compared with studies in older individuals. Our study does have some limitations. Although DBS biospecimens were all obtained from newborns in California, we did not match DS and non-DS newborns by demographic variables such as sex, race/ethnicity, or birth year. This should not have biased our findings, however, as our EWAS was adjusted for sex and principal components, and similar results were found in Latinos and non-Latino whites. Second, analytical tools such as ReFACTor and cell-type deconvolution were developed in euploid individuals, although we did confirm some known differences in blood cell proportions (using conventional cell enumeration methods) between DS and non-DS individuals. Reference-free adjustment for cell-type composition was performed in our EWAS, given the highly significant differences in estimated blood cell proportions and to maximize our power to detect epigenetic changes associated with trisomy 21 ; however, we cannot rule out that some of the DNA methylation changes associated with DS may reflect differences in peripheral blood cell composition between DS and non-DS newborns, and future studies should explore the epigenetic effects of DS in sorted blood cells. Finally, our study was limited to newborn wholeblood samples, and would not detect tissue-specific DNA methylation differences outside of blood that may underlie DSrelated phenotypes. Studies have, however, demonstrated similarities in the epigenetic effects of T21 across tissues ${ }^{40}$, and in the use of blood DNA methylation as a biomarker for traits in other tissues, such as brain-related phenotypes ${ }^{43}$.

Our results demonstrate the profound genome-wide effects of T21 on DNA methylation, with important implications for the defects in hematopoiesis, cognition, immune function, and other developmental processes that arise in individuals with DS. Determining the etiologies of these epigenetic changes will be essential to understand and potentially ameliorate DS phenotypes. Epigenetic changes in DS may occur due to triplication of specific genes on Hsa21, such as $H M G N 1^{23}$ or $D N M T 3 L^{55}$, the effects of additional genomic material on three-dimensional chromatin organization, or via some compensatory mechanism triggered early in DS fetal development. One might predict compensatory hypermethylation of triplicated genes; thus, it is also important to understand why Hsa21 is largely hypomethylated in DS, and how this hypomethylation is distributed across the three copies of Hsa21. Finally, case-control studies within DS populations are required to investigate the association between epigenetic variation across tissues and the variable penetrance and expressivity of DS-related phenotypes.

\section{Methods}

Study subjects. This study was approved by Institutional Review Boards at the California Health and Human Services Agency, University of Southern California, and University of California Berkeley, and by Hammersmith and Queen Charlotte's Hospital Research Ethics Committee (ref 04/Q0406/145). The deidentified 
newborn DBS from the California Biobank Program for this project (SIS request numbers 572 and 600) were obtained with a waiver of consent from the Committee for the Protection of Human Subjects of the State of California. FL samples were obtained with written consent.

DBS were obtained from 198 DS newborns, without a leukemia diagnosis by 15 years of age, from the California Biobank Program via linkage between the California Department of Public Health Genetic Disease Screening Program and California Cancer Registry ${ }^{50}$. We also obtained newborn DBS from 442 non-DS (cancer-free) children from the California Biobank Program ${ }^{56}$. Demographic and birth-related data for subjects that passed QC are summarized in Table 1. The majority of individuals were reported as Latino $(N=357)$ or non-Latino white $(N=178)$, and the remainder as African American $(N=34)$, Asian/Pacific Islander $(N=55)$, or other $(N=11)$

A separate sample set was used for gene expression studies. Second-trimester FL samples were collected during elective surgical termination of pregnancy and processed immediately. Donated fetal tissue was also provided by the Human Developmental Biology Resource (www.hdbr.org) regulated by the UK Human Tissue Authority (www.hta.gov.uk).

Genome-wide DNA methylation arrays. DNA was extracted from one-third portions of each newborn DBS using the Qiagen DNA Investigator blood card protocol, and bisulfite conversion performed using Zymo EZ DNA Methylation kits. Bisulfite-converted DNA samples from DS and non-DS newborns were blockrandomized (ensuring equivalent distribution of sex and race/ethnicity on all plates) and run on Illumina Infinium MethylationEPIC Beadchip genome-wide DNA methylation arrays.

DNA methylation array data processing, visualization, and annotation. For QC assessment of DNA methylation array data, we imported raw IDAT files into R and used the "minfi" package to calculate mean detection $P$ values using the "detectionP" function. Further data QC and normalization were performed using the R package "SeSAMe," with background correction using "noob" and using $P$ value with out-ofband $(\mathrm{OOB})$ array hybridization for removal of poor-performing probes, accounting for deleted and hyperpolymorphic regions ( $\mathrm{R}$ version: 3.6 .0$)^{57,58}$. The $\mathrm{R}$ package "conumee" was used to generate copy-number variation (CNV) plots for all subjects to check T21 status ${ }^{59}$, with twenty randomly selected non-DS newborns used to construct a CNV reference, and subjects were removed if the reported DS status did not match T21 status based on visual inspection (one "control" appeared to have T21 and was excluded). Subjects with missingness $>5 \%$ ( 2 DS and 2 non-DS) were removed, resulting in a final study number of 196 DS and 439 non-DS newborns. CpG probes with missingness $>5 \%$ were subsequently removed $(N=137,060)$, and the remaining missing values imputed using "impute.knn" function from the "impute" package.

We removed probes located on chromosomes $\mathrm{X}$ and $\mathrm{Y}$ as well as CpGs located at SNP sites with a minor allelic frequency $>5 \%$, resulting in a final CpG probe set $N=651,772$. Using data from this final set of probes, and also excluding probes on Hsa21, we calculated PCs in R using the "prcomp" command to create PCA plots. Additional dimensional reduction plots to visualize clustering within samples were generated using the t-SNE algorithm, with the "Rtsne" package ${ }^{60}$. Betas were converted to $M$ values for construction of heatmaps using the "ComplexHeatmap" package $^{61}$ for the $2000 \mathrm{CpGs}$ with the greatest mean absolute deviation across chromosomes, excluding $21, \mathrm{X}$, and $\mathrm{Y}$, and annotated for DS status, sex, and deconvoluted blood cell proportions.

All CpGs in the EWAS results were annotated using the annotation database from the "IlluminaHumanMethylationEPICanno.ilm10b4.hg19" package in $\mathrm{R}^{62}$. DMRs of interest were visualized using the "coMET" package ${ }^{63}$.

\section{Assessment and adjustment of cell-type heterogeneity. Reference-based} deconvolution of blood cell proportions in DS and non-DS newborns was performed using the Identifying Optimal Libraries algorithm ${ }^{64,65}$. We used the "estimateCellCounts2" function in the R package "FlowSorted.Blood.EPIC" and DNA methylation data from cord blood cell reference samples in the R package "FlowSorted.CordBloodCombined.450k," to estimate proportions of monocytes, granulocytes, natural killer cells, B lymphocytes, T lymphocytes (both $\mathrm{CD} 4^{+}$and $\mathrm{CD} 8^{+}$), and $\mathrm{nRBC} /$ erythroblasts in newborns $\mathrm{s}^{66,67}$. We performed separate linear regression tests with each blood cell-type proportion as the dependent variable and DS status as the independent variable, adjusting for plate, sex, the first ten EPISTRUCTURE PCs to account for genetic ancestry (see below), gestational age, age at DBS collection, and birth weight. We also tested the association of each blood cell-type proportion with birth-related and demographic variables. Within DS newborns, we performed additional regression models to test the association between GATA1 mutations, treated either as a binary variable (presence/absence) or a linear variable (i.e., VAF), and blood cell proportions and birth variables, adjusting for plate, sex, and EPISTRUCTURE PCs as above.

To account for cell-type heterogeneity in the EWAS, we obtained ReferenceFree Adjustment for Cell-Type composition (ReFACTor) PCs using the GLINT tool (v1.0.4) ${ }^{68,69}$, with the assumed number of cell types in the data (k) set to 7 to align with the reference-based approach described above, and with adjustment for plate and sex.
GATA1 mutation sequencing in DS newborns. We performed targeted sequencing of GATA1 in 184 DS newborns with sufficiently remaining DNA isolated from DBS, using methods modified from previously described protocols 8 ,70. In brief, targeted amplification of GATA1 from isolated genomic DNA was performed in tandem, with the addition of sample barcodes and sequencing adapters. Six primer pairs generating 150-210-bp amplicons covering the entirety of exon 2 and the first 115 bases of exon 3 and including three exon/intron boundaries were individually amplified in quadruplicate following Fluidigm's Access Array ${ }^{\mathrm{TM}}$ IFC 4-Primer Amplicon Tagging Workflow (Fluidigm: PN 68000161). Primer sequences are included in Supplementary Table 4. After amplification, $2 \mu \mathrm{l}$ for each sample were pooled and PCR products purified using AMPureXP beads (Beckman Coulter). Quality and size distribution were determined using a Tapestation system (Agilent Technologies). Library concentration was determined by the Qubit dsDNA HS Assay kit (Thermo Fisher). Sequencing was performed on an Illumina MiSeq as 150 base-paired-end reads. Mapping and variant analysis were performed using an in-house pipeline generating VarScan somatic data ${ }^{71}$, as previously described ${ }^{8,70}$. VAF were manually verified and compared to in-run controls using the Integrative Genomics Viewer for visualization ${ }^{72}$. The limit of detection of mutant GATA1 sequence was $0.3-2 \%$ depending on read quality and depth of sequencing.

Epigenome-wide association analyses. GLINT was used to obtain EPISTRUCTURE PCs, adjusting for the first ten ReFACTor PCs, to account for genetic ancestry ${ }^{73}$. CpG probes on chromosomes X and Y, and at SNP sites with MAF > $5 \%$ were removed, resulting in a final probe set $N=651,772$. A multiethnic EWAS of DS was performed using linear regression in $\mathrm{R}$, with each $\mathrm{CpG} \beta$-value as the dependent variable and DS status the independent variable, adjusting for sex, plate, the first ten ReFACTor PCs to adjust for cell-type proportions, and first ten EPISTRUCTURE PCs to adjust for genetic ancestry.

For sensitivity analysis to account for potential confounding of uncorrected population stratification in the overall multiethnic EWAS, we repeated the EWAS separately in the two largest self-reported race/ethnicity groups, in Latinos (DS $N=104$, non-DS $N=256$ ) and non-Latino whites (DS $N=54$, non-DS $N=124$ ). In these race/ethnicity-stratified EWAS models, we only adjusted for the first six EPISTRUCTURE PCs to reach acceptable levels of epigenomic inflation $(\lambda=1.46$ for Latinos and 1.09 for non-Latino whites). In addition, we repeated the multiethnic EWAS following: (1) removal of the separate cluster of DS individuals $(N=34)$ observed in our visualization plots and with high nRBC proportions in the cell-type deconvolution analyses (Figs. 1 and 2), or (2) removal of DS newborns with any somatic GATA1 mutations identified by sequencing $(N=30)$.

To explore epigenetic changes associated with GATA1 mutations, we performed a separate EWAS within DS newborns with GATA1 mutation status as a binary dependent variable. Bonferroni correction was applied to correct for multiple testing $\left(P<7.67 \times 10^{-8}\right.$, based on $\left.651,772 \mathrm{CpGs}\right)$.

Gene pathway enrichment analyses were performed for genes overlapped by epigenome-wide significant CpGs using the "methylglm" function in the R package "methylGSA," with assessment of GO and KEGG pathways ${ }^{74}$. We used a gene-list minimum size of 10 and maximum size of 500, and only considered pathways with an FDR-corrected $P$ value $<0.05$ as significant.

We also performed enrichment analysis to assess the significant overlap between epigenome-wide significant $\mathrm{CpGs}$ and genomic locations (i.e., promoters, exons, introns, $1-5 \mathrm{~Kb}, 3^{\prime}$-UTRs, $5^{\prime}$-UTRs, intergenic, intron/exon boundaries, $\mathrm{CpG}$ islands, shelves, shores, and open sea), as well as functional features, including TF-binding sites, histone modification markers, DHS, and predicted enhancer regions. The number of significant and nonsignificant $\mathrm{CpGs}$ overlapping each feature was compared by the Fisher's exact test. For TF-binding sites, we included all available TFs $(N=161)$ in the ENCODE ChiP-seq database for the K562 cell line (wgEncodeRegTfbsClusteredV3.bed). Histone modification data were downloaded for primary HSCs (cell line E035) from the Roadmap Epigenomics Mapping Consortium database ${ }^{75}$. DHS sites were downloaded from the ENCODE project (wgEncodeRegDnaseClusteredV3.bed file). Finally, we assessed overlap with previously identified enhancer regions for three HSC cell lines (BI_CD34_Primary_RO01536, BI_CD34_Primary_RO01480, and BI_CD34_Primary_RO01549), CD19+ B cells (CD19_primary), GM12878 lymphoblastoid cells, K562 cells, and four brain cell lines (astrocytes, frontal lobe cells, temporal lobe cells, and hippocampus cells) ${ }^{76}$. All enrichment analyses were performed separately for hyper- and hypomethylated CpGs, and Bonferroni correction for multiple testing was applied as warranted for each analysis based on the number of comparisons.

DMRs associated with DS were identified using two different methods, DMRcate and comb- $\mathrm{p}^{77,78}$. The $P$ values obtained in each EWAS were used for comb-p. DMRcate was run with adjustment for cell-type heterogeneity using the first ten ReFACTor PCs, as well as for sex, plate, and the first ten EPISTRUCTURE PCs (except in race/ethnicity-stratified analyses below). We retained DMRs that spanned a minimum of two CpGs, had a maximum distance of $1000 \mathrm{bp}$ between methylation peaks, had an FDR-corrected $P$ value $<0.01$ in DMRcate, had a Šidákcorrected $P$ value $<0.01$ in comb-p, and that displayed any overlap between the coordinates of regions called by DMRcate and comb-p.

In the analyses of DMRs in DS, we generated DMR calls for (i) overall DS versus non-DS subjects, (ii) Latino and (iii) non-Latino white-stratified analyses (in both of which the first six EPISTRUCTURE PCs were used to adjust for genetic 
ancestry, as for the EWAS models above), (iv) following removal of the 34 DS newborns with high nRBC proportions, (v) following removal of the 30 DS newborns with GATA1 mutations, and (vi) for GATA1 mutation status as described for the EWAS of GATA1 mutations. To assess the potential functions of genes overlapped by the most significant DMRs in the overall analysis of DS, we investigated the presence of SNPs, and their corresponding trait associations, in the NHGRI-EBI Catalog of published GWAS and with reported $P$ values $<5.0 \times 10^{-8}$ in regions spanning +50 and $-50 \mathrm{~Kb}$ of each DMR locus ${ }^{79}$.

\section{Gene expression analysis in DS and non-DS FL CD34+ cells by bulk RNA}

sequencing. GATA1 mutation analysis, CD34+ separation, and immunohistochemistry were performed as previously described ${ }^{3,80}$. Fluorescence in situ hybridization was used to confirm the presence $(N=3)$ or absence $(N=3)$ of T21 in FL. Bulk RNA sequencing of DS and non-DS FL cells was performed using the SMARTSeq2 protocol $^{81}$. In brief, 100 purified HSC or progenitor cells (HSPCs) from gestation-matched DS $(N=3)$ and non-DS $(N=3)$ 2nd-trimester FL samples were sorted directly into lysis buffer containing $0.4 \%$ Triton X-100 (Sigma-Aldrich), RNase inhibitor (Clontech), 2.5-mM dNTPs (Thermo Fisher), and $2.5-\mu \mathrm{M}$ oligo-dT30VN primer (Biomers.net). cDNA was generated using SuperScript II (Invitrogen), preamplified using KAPA HiFi HotStart ReadyMix (KAPA Biosystems) using 18 cycles of amplification. After PCR amplification, the cDNA libraries were purified with AMPure XP beads (Beckman Coulter) according to the manufacturer's instructions. Post-purification libraries were resuspended in EB buffer (Qiagen). The quality of cDNA traces was assessed by using a High Sensitivity DNA Kit in a Bioanalyzer instrument (Agilent Technologies). Library preparation was performed using the Nextera XT DNA Library Preparation Kit (Illumina) according to the manufacturer's instructions. Indexed cDNA libraries were multiplexed and sequenced using Illumina HiSeq2500 to generate 150 -bp paired-end reads, yielding $>30$ million reads per sample.

Following sequencing, QC analysis was conducted using the fastQC package (http://www.bioinformatics.babraham.ac.uk/projects/fastqc). Reads were mapped to the human genome assembly hg19 using STAR software ${ }^{82}$. Quality and adapter trimming were performed using TrimGalore (https://github.com/FelixKrueger/ TrimGalore). The featureCounts function from the Subread package in $\mathrm{R}$ was used to quantify gene expression levels using standard parameters. We excluded from our analyses any genes with $<100$ combined reads across all six samples. Differential gene expression between groups was assessed using the DESeq2 package ${ }^{83}$.

Single-cell gene expression analysis in DS and non-DS FL CD34+ cells. Singlecell analysis of RUNX1 and FLI1 gene expression was performed by reverse transcription (RT)-qPCR using the Biomark HD microfluidics system (Fluidigm). Flow cytometry was performed as previously described ${ }^{84}$. Samples were FACSsorted using BD Fusion instruments, and data analyzed using FlowJo software. Single cells from FL common myeloid progenitors (CMP:Lin-CD34+CD38+ CD45RA-CD123+) and megakaryocyte-erythroid progenitors (MEP:LinCD34+CD38+CD45RA-CD123-) from three DS and three non-DS subjects were index-sorted into a 96-well plate containing $5 \mu \mathrm{L}$ of preamplification mix, which contained One-Step RT-PCR System with Platinum Taq kit (Invitrogen), SUPERASE-In RNase inhibitor (Ambion), low EDTA TE buffer (Invitrogen), and $0.2 \mathrm{X}$ Taqman assay mastermix. Plates were sealed, briefly centrifuged, and cDNA synthesis and sequence-specific preamplification performed (three-step PCR of step 1, reverse transcriptase at $50^{\circ} \mathrm{C}$ for $15 \mathrm{~min}$, step 2, inactivation of RTase, activation of Taq at $95^{\circ} \mathrm{C}$ for $2 \mathrm{~min}$, and step 3, specific target amplification at $95^{\circ} \mathrm{C}$ for $15 \mathrm{~s}$, then $60^{\circ} \mathrm{C}$ for $4 \mathrm{~min}$ repeated for 20 cycles). Preamplified products were diluted by adding $20 \mu \mathrm{L}$ of low EDTA TE buffer, and samples then analyzed using Universal PCR Master Mix (Applied Biosystems) and individual Taqman gene expression assays (RUNX1 Assay ID: Hs01021970_m1, FLI1 Assay ID: Hs00956711_m1 [Life Technologies]), on the Biomark System (Fluidigm) using the 96.96 Dynamic Arrays as per the manufacturer's protocol. Sorted cells were simultaneously analyzed for relative expression levels of RUNX1 and FLI1. Gene expression was normalized to the average expression of three housekeeping genes B2M (Assay ID: Hs00984230_m1), GAPDH (Hs02758991_g1), and ACTB (Assay ID Hs01060665_g1 $)^{84}$.

Reporting summary. Further information on research design is available in the Nature Research Reporting Summary linked to this article.

\section{Data availability}

ENCODE TF-binding site dataset wgEncodeRegTfbsClusteredV3.bed.gz was downloaded from the UCSC Genome Browser (https://hgdownload.cse.ucsc.edu/goldenpath/hg19/ encodeDCC/wgEncodeRegTfbsClustered/). Histone modification data were downloaded for primary HSCs (cell line E035, CD34 primary cells) from the Roadmap Epigenomics Mapping Consortium database (https://egg2.wustl.edu/roadmap/data/byFileType/peaks/). ENCODE DNase I hypersensitive site data are available at http://hgdownload.cse.ucsc.edu/ goldenpath/hg19/encodeDCC/wgEncodeRegDnaseClustered/. NHGRI-EBI GWAS Catalog data are available at https://www.ebi.ac.uk/gwas/docs/file-downloads. FLI1 transcript variant data were downloaded from the BLUEPRINT Consortium Blood Atlas (https://blueprint. haem.cam.ac.uk/mRNA/). This study used biospecimens from the California Biobank
Program. Any uploading of genomic data (including genome-wide DNA methylation data) and/or sharing of these biospecimens or individual data derived from these biospecimens has been determined to violate the statutory scheme of the California Health and Safety Code Sections 124980(j), 124991(b), (g), (h), and 103850 (a) and (d), which protect the confidential nature of biospecimens and individual data derived from biospecimens. The individual-level data derived from these biospecimens and that support the findings of this study are available from the corresponding author upon request, and with permission from the California Biobank Program. RNA-seq data from DS and non-DS FL CD34+ cells have been deposited at the Gene Expression Omnibus (GEO) with accession code: GSE160637. Source data are provided with this paper.

Received: 20 August 2020; Accepted: 5 January 2021; Published online: 05 February 2021

\section{References}

1. Parker, S. E. et al. Updated national birth prevalence estimates for selected birth defects in the United States, 2004-2006. Birth Defects Res. A. Clin. Mol. Teratol. 88, 1008-1016 (2010).

2. Korenberg, J. R. et al. Down syndrome phenotypes: the consequences of chromosomal imbalance. Proc. Natl. Acad. Sci. U. S. A. 91, 4997-5001 (1994)

3. Roy, A. et al. Perturbation of fetal liver hematopoietic stem and progenitor cell development by trisomy 21. Proc. Natl Acad. Sci. U. S. A. 109, 17579-17584 (2012).

4. de Hingh, Y. C. et al. Intrinsic abnormalities of lymphocyte counts in children with down syndrome. J. Pediatr. 147, 744-747 (2005).

5. Ram, G. \& Chinen, J. Infections and immunodeficiency in Down syndrome. Clin. Exp. Immunol. 164, 9-16 (2011).

6. Hasle, H., Clemmensen, I. H. \& Mikkelsen, M. Risks of leukaemia and solid tumours in individuals with Down's syndrome. Lancet 355, 165-169 (2000).

7. Hasle, H., Friedman, J. M., Olsen, J. H. \& Rasmussen, S. A. Low risk of solid tumors in persons with Down syndrome. Genet. Med. 18, 1151-1157 (2016).

8. Roberts, I. et al. GATA1-mutant clones are frequent and often unsuspected in babies with Down syndrome: identification of a population at risk of leukemia. Blood 122, 3908-3917 (2013).

9. Bhatnagar, N., Nizery, L., Tunstall, O., Vyas, P. \& Roberts, I. Transient abnormal myelopoiesis and AML in Down Syndrome: an update. Curr. Hematol. Malig. Rep. 11, 333-341 (2016).

10. Klusmann, J. H. et al. Treatment and prognostic impact of transient leukemia in neonates with Down syndrome. Blood 111, 2991-2998 (2008).

11. Antonarakis, S. E. Down syndrome and the complexity of genome dosage imbalance. Nat. Rev. Genet. 18, 147-163 (2017).

12. Letourneau, A. et al. Domains of genome-wide gene expression dysregulation in Down's syndrome. Nature 508, 345-350 (2014)

13. Liu, B., Filippi, S., Roy, A. \& Roberts, I. Stem and progenitor cell dysfunction in human trisomies. EMBO Rep. 16, 44-62 (2015)

14. Prandini, P. et al. Natural gene-expression variation in Down syndrome modulates the outcome of gene-dosage imbalance. Am. J. Hum. Genet. 81, 252-263 (2007).

15. Kerkel, K. et al. Altered DNA methylation in leukocytes with trisomy 21. PLoS Genet. 6, e1001212 (2010)

16. Bacalini, M. G. et al. Identification of a DNA methylation signature in blood cells from persons with Down Syndrome. Aging 7, 82-96 (2015).

17. Henneman, P. et al. Widespread domain-like perturbations of DNA methylation in whole blood of Down syndrome neonates. PLoS ONE 13, e0194938 (2018).

18. Mendioroz, M. et al. Trans effects of chromosome aneuploidies on DNA methylation patterns in human Down syndrome and mouse models. Genome Biol. 16, 263 (2015)

19. Sailani, M. R. et al. DNA-methylation patterns in trisomy 21 using cells from monozygotic twins. PLoS ONE 10, e0135555 (2015)

20. Thilaganathan, B., Tsakonas, D. \& Nicolaides, K. Abnormal fetal immunological development in Down's syndrome. Br. J. Obstet. Gynaecol. 100, 60-62 (1993).

21. Jung, I. et al. A compendium of promoter-centered long-range chromatin interactions in the human genome. Nat. Genet. 51, 1442-1449 (2019).

22. Grassi, L. et al. Cell type specific novel lncRNAs and circRNAs in the BLUEPRINT haematopoietic transcriptomes atlas. Haematologica https://doi. org/10.3324/haematol.2019.238147 (2020).

23. Lane, A. A. et al. Triplication of a $21 \mathrm{q} 22$ region contributes to B cell transformation through HMGN1 overexpression and loss of histone H3 Lys27 trimethylation. Nat. Genet. 46, 618-623 (2014).

24. Chen, M. J., Yokomizo, T., Zeigler, B. M., Dzierzak, E. \& Speck, N. A. Runx1 is required for the endothelial to haematopoietic cell transition but not thereafter. Nature 457, 887-891 (2009). 
25. Kubota, Y. et al. Integrated genetic and epigenetic analysis revealed heterogeneity of acute lymphoblastic leukemia in Down syndrome. Cancer Sci. 110, 3358-3367 (2019).

26. Miyoshi, H. et al. Alternative splicing and genomic structure of the AML1 gene involved in acute myeloid leukemia. Nucleic Acids Res. 23, 2762-2769 (1995).

27. Bee, T. et al. Nonredundant roles for Runxl alternative promoters reflect their activity at discrete stages of developmental hematopoiesis. Blood 115, 3042-3050 (2010).

28. Sroczynska, P., Lancrin, C., Kouskoff, V. \& Lacaud, G. The differential activities of Runx1 promoters define milestones during embryonic hematopoiesis. Blood 114, 5279-5289 (2009).

29. Lie-A-Ling, M. et al. Regulation of RUNX1 dosage is crucial for efficient blood formation from hemogenic endothelium. Development 145, https://doi.org/ 10.1242/dev.149419 (2018).

30. Cai, Z. et al. Haploinsufficiency of AML1 affects the temporal and spatial generation of hematopoietic stem cells in the mouse embryo. Immunity 13, 423-431 (2000).

31. Webber, B. R. et al. DNA methylation of Runx1 regulatory regions correlates with transition from primitive to definitive hematopoietic potential in vitro and in vivo. Blood 122, 2978-2986 (2013).

32. Bastian, L. S., Kwiatkowski, B. A., Breininger, J., Danner, S. \& Roth, G. Regulation of the megakaryocytic glycoprotein IX promoter by the oncogenic Ets transcription factor Fli-1. Blood 93, 2637-2644 (1999).

33. Hart, A. et al. Fli-1 is required for murine vascular and megakaryocytic development and is hemizygously deleted in patients with thrombocytopenia. Immunity 13, 167-177 (2000).

34. Eisbacher, M. et al. Protein-protein interaction between Fli-1 and GATA-1 mediates synergistic expression of megakaryocyte-specific genes through cooperative DNA binding. Mol. Cell. Biol. 23, 3427-3441 (2003).

35. Huang, H. et al. Differentiation-dependent interactions between RUNX-1 and FLI-1 during megakaryocyte development. Mol. Cell. Biol. 29, 4103-4115 (2009).

36. Pimkin, M. et al. Divergent functions of hematopoietic transcription factors in lineage priming and differentiation during erythro-megakaryopoiesis. Genome Res. 24, 1932-1944 (2014).

37. Raslova, H. et al. FLI1 monoallelic expression combined with its hemizygous loss underlies Paris-Trousseau/Jacobsen thrombopenia. J. Clin. Invest. 114, 77-84 (2004).

38. Stockley, J. et al. Enrichment of FLI1 and RUNX1 mutations in families with excessive bleeding and platelet dense granule secretion defects. Blood $\mathbf{1 2 2}$ 4090-4093 (2013).

39. Bergiers, I. et al. Single-cell transcriptomics reveals a new dynamical function of transcription factors during embryonic hematopoiesis. Elife 7, https://doi. org/10.7554/eLife.29312 (2018).

40. Laufer, B. I., Hwang, H., Vogel Ciernia, A., Mordaunt, C. E. \& LaSalle, J. M. Whole genome bisulfite sequencing of Down syndrome brain reveals regional DNA hypermethylation and novel disorder insights. Epigenetics 14, 672-684 (2019).

41. Fukui, H., Runker, A., Fabel, K., Buchholz, F. \& Kempermann, G. Transcription factor Runx1 is pro-neurogenic in adult hippocampal precursor cells. PLoS ONE 13, e0190789 (2018).

42. Theriault, F. M. et al. Role for Runxl in the proliferation and neuronal differentiation of selected progenitor cells in the mammalian nervous system. J. Neurosci. 25, 2050-2061 (2005).

43. Jia, T. et al. Epigenome-wide meta-analysis of blood DNA methylation and its association with subcortical volumes: findings from the ENIGMA Epigenetics Working Group. Mol. Psychiatry, https://doi.org/10.1038/s41380-019-0605-Z (2019).

44. Bakircioglu, M. et al. The essential role of centrosomal NDE1 in human cerebral cortex neurogenesis. Am. J. Hum. Genet. 88, 523-535 (2011).

45. Ishibashi, M. et al. Targeted disruption of mammalian hairy and Enhancer of split homolog-1 (HES-1) leads to up-regulation of neural helix-loop-helix factors, premature neurogenesis, and severe neural tube defects. Genes Dev. 9, 3136-3148 (1995).

46. Inoue, M. et al. Prdm8 regulates the morphological transition at multipolar phase during neocortical development. PLOS ONE 9, e86356 (2014).

47. Webb, D., Roberts, I. \& Vyas, P. Haematology of Down syndrome. Arch. Dis. Child. Fetal Neonatal Ed. 92, F503-F507 (2007).

48. Nitzan, I. et al. Elevated nucleated red blood cells in neonates with Down syndrome and pulmonary hypertension. J. Pediatr. 213, 232-234 (2019).

49. Bozner, P. Transient myeloproliferative disorder with erythroid differentiation in Down syndrome. Arch. Pathol. Lab. Med. 126, 474-477 (2002).

50. Brown, A. L. et al. Inherited genetic susceptibility of acute lymphoblastic leukemia in Down syndrome. Blood 134, 1227-1237 (2019).

51. Kim, N. \& Jinks-Robertson, S. Transcription as a source of genome instability. Nat. Rev. Genet. 13, 204-214 (2012).
52. Azad, P. et al. Senp1 drives hypoxia-induced polycythemia via GATA1 and Bcl-xL in subjects with Monge's disease. J. Exp. Med. 213, 2729-2744 (2016)

53. Debieve, F., Moiset, A., Thomas, K., Pampfer, S. \& Hubinont, C. Vascular endothelial growth factor and placenta growth factor concentrations in Down's syndrome and control pregnancies. Mol. Hum. Reprod. 7, 765-770 (2001).

54. Silver, M. J. et al. Independent genomewide screens identify the tumor suppressor VTRNA2-1 as a human epiallele responsive to periconceptional environment. Genome Biol. 16, 118 (2015).

55. Lu, J. et al. Global hypermethylation in fetal cortex of Down syndrome due to DNMT3L overexpression. Hum. Mol. Genet. 25, 1714-1727 (2016).

56. Nielsen, A. B. et al. Increased neonatal level of arginase 2 in cases of childhood acute lymphoblastic leukemia implicates immunosuppression in the etiology. Haematologica 104, e514-e516 (2019).

57. Zhou, W., Triche, T. J. Jr, Laird, P. W. \& Shen, H. SeSAMe: reducing artifactual detection of DNA methylation by Infinium BeadChips in genomic deletions. Nucleic Acids Res. 46, e123 (2018).

58. Triche, T. J. Jr, Weisenberger, D. J., Van Den Berg, D., Laird, P. W. \& Siegmund, K. D. Low-level processing of Illumina Infinium DNA Methylation BeadArrays. Nucleic Acids Res 41, e90 (2013).

59. Mah, C. K., Mesirov, J. P. \& Chavez, L. An accessible GenePattern notebook for the copy number variation analysis of Illumina Infinium DNA methylation arrays. F1000Res 7, https://doi.org/10.12688/f1000research.16338.1 (2018).

60. van der Maaten, L. J. P. \& Hinton, G. E. Visualizing high-dimensional data using t-SNE. J. Mach. Learn. Res. 9, 2579-2605 (2008).

61. Gu, Z., Eils, R. \& Schlesner, M. Complex heatmaps reveal patterns and correlations in multidimensional genomic data. Bioinformatics 32, 2847-2849 (2016).

62. Hansen, K. D. IlluminaHumanMethylationEPICanno.ilm10b2.hg19: annotation for Illumina's EPIC methylation arrays. R package version 0.6.0, https://bitbucket.com/kasperdanielhansen/Illumina_EPIC (2016).

63. Martin, T. C., Yet, I., Tsai, P. C. \& Bell, J. T. coMET: visualisation of regiona epigenome-wide association scan results and DNA co-methylation patterns. BMC Bioinform. 16, 131 (2015).

64. Gervin, K. et al. Systematic evaluation and validation of reference and library selection methods for deconvolution of cord blood DNA methylation data. Clin. Epigenetics 11, 125 (2019).

65. Koestler, D. C. et al. Improving cell mixture deconvolution by identifying optimal DNA methylation libraries (IDOL). BMC Bioinform. 17, 120 (2016).

66. Salas, L. A. \&. Koestler, D. C. Illumina EPIC data on immunomagnetic sorted peripheral adult blood cells. R package version 1.5.3, https://github.com/ immunomethylomics/FlowSorted.Blood.EPIC (2019).

67. Bakulski, K. M. et al. DNA methylation of cord blood cell types: applications for mixed cell birth studies. Epigenetics 11, 354-362 (2016).

68. Rahmani, E. et al. Sparse PCA corrects for cell type heterogeneity in epigenome-wide association studies. Nat. Methods 13, 443-445 (2016).

69. Rahmani, E. et al. GLINT: a user-friendly toolset for the analysis of highthroughput DNA-methylation array data. Bioinformatics 33, 1870-1872 (2017).

70. Labuhn, M. et al. Mechanisms of progression of myeloid preleukemia to transformed myeloid leukemia in children with Down syndrome. Cancer Cell. 36, 123-138.e10 (2019)

71. Koboldt, D. C. et al. VarScan: variant detection in massively parallel sequencing of individual and pooled samples. Bioinformatics 25, 2283-2285 (2009).

72. Robinson, J. T., Thorvaldsdottir, H., Wenger, A. M., Zehir, A. \& Mesirov, J. P. Variant review with the integrative genomics viewer. Cancer Res 77, e31-e34 (2017).

73. Rahmani, E. et al. Genome-wide methylation data mirror ancestry information. Epigenetics Chromatin 10, 1 (2017).

74. Ren, X. \& Kuan, P. F. methylGSA: a Bioconductor package and Shiny app for DNA methylation data length bias adjustment in gene set testing. Bioinformatics 35, 1958-1959 (2019).

75. Bernstein, B. E. et al. The NIH Roadmap Epigenomics Mapping Consortium. Nat. Biotechnol. 28, 1045-1048 (2010).

76. Hnisz, D. et al. Super-enhancers in the control of cell identity and disease. Cell 155, 934-947 (2013).

77. Peters, T. J. et al. De novo identification of differentially methylated regions in the human genome. Epigenetics Chromatin 8, 6 (2015).

78. Pedersen, B. S., Schwartz, D. A., Yang, I. V. \& Kechris, K. J. Comb-p: software for combining, analyzing, grouping and correcting spatially correlated P-values. Bioinformatics 28, 2986-2988 (2012).

79. Buniello, A. et al. The NHGRI-EBI GWAS catalog of published genome-wide association studies, targeted arrays and summary statistics 2019. Nucleic Acids Res. 47, D1005-D1012 (2019).

80. Tunstall-Pedoe, O. et al. Abnormalities in the myeloid progenitor compartment in Down syndrome fetal liver precede acquisition of GATA1 mutations. Blood 112, 4507-4511 (2008).

81. Picelli, S. et al. Full-length RNA-seq from single cells using Smart-seq2. Nat Protoc. 9, 171-181 (2014). 
82. Dobin, A. et al. STAR: ultrafast universal RNA-seq aligner. Bioinformatics 29, 15-21 (2013)

83. Love, M. I., Huber, W. \& Anders, S. Moderated estimation of fold change and dispersion for RNA-seq data with DESeq2. Genome Biol. 15, 550 (2014).

84. O'Byrne, S. et al. Discovery of a CD10-negative B-progenitor in human fetal life identifies unique ontogeny-related developmental programs. Blood 134, 1059-1071 (2019).

85. Olsen, I. E., Groveman, S. A., Lawson, M. L., Clark, R. H. \& Zemel, B. S. New intrauterine growth curves based on United States data. Pediatrics 125, e214-e224 (2010).

\section{Acknowledgements}

This work was supported by an Alex's Lemonade Stand Foundation "A" Award (A.J.d.S.), a National Institutes of Health (NIH) National Cancer Institute (NCI) Grant R01CA175737 (J.L.W. and X.M.), and a NIH NCI Administrative Supplement grant 3R01CA175737-05S1 (J.L.W., X.M., and A.J.d.S.). A.R. is supported by a Blood Cancer UK Clinician Scientist Fellowship (17001), Lady Tata Memorial International Fellowship, and Wellcome Clinical Research Career Development Fellowship (216632/Z/19/Z). P.V. and I.R. are supported by Blood Cancer UK Specialist Programme Grant 13001 and by the NIHR Oxford Biomedical Centre Research Fund. The content of this manuscript is solely the responsibility of the authors and does not necessarily represent the official views of the National Institutes of Health. A subset of biospecimens and/or data used in this study were obtained from the California Biobank Program at the California Department of Public Health (CDPH), SIS request numbers 572 and 600, in accordance with Section 6555(b), 17 CCR. The CDPH is not responsible for the results or conclusions drawn by the authors of this publication. We thank Robin Cooley and Steve Graham (Genetic Disease Screening Program, CDPH) for their assistance and expertise in the procurement and management of DBS specimens. We thank Hong Quach and Diana Quach at the UC Berkeley QB3 Genetic Epidemiology and Genomics Laboratory for their support in preparing and processing samples for genome-wide DNA methylation arrays. Human fetal material was provided by the Joint MRC/Wellcome Trust Grant 099175/Z/12/Z Human Developmental Biology Resource (http://hdbr.org). We would like to acknowledge the WIMM Flow Cytometry Facility, the High-Throughput Genomics Group at the Wellcome Trust Centre for Human Genetics; and MRC WIMM Single Cell Facility.

\section{Author contributions}

A.J.d.S., J.L.W., X.M., A.R. and I.R. designed the study. I.S.M., T.J., K.M.W., A.R., I.R., J.L.W. and A.J.d.S. prepared the paper. N.E., A.R., H.M.H., S.S.M., J.A. and K.G. performed experiments. I.S.M., S.L., T.J., N.E., H.M.H., P.P., J.M.S., R.R., K.D.S., K.M.W., A.R., I.R. and A.J.d.S. analyzed data. J.L.W., X.M., P.V. and I.R. provided study samples. All authors edited and approved the paper.

\section{Competing interests}

The authors declare no competing interests.

\section{Additional information}

Supplementary information The online version contains supplementary material available at https://doi.org/10.1038/s41467-021-21064-z.

Correspondence and requests for materials should be addressed to A.J.d.S.

Peer review information Nature Communications thanks Jeffrey Craig, and the other, anonymous, reviewer for their contribution to the peer review of this work. Peer reviewer reports are available.

Reprints and permission information is available at http://www.nature.com/reprints

Publisher's note Springer Nature remains neutral with regard to jurisdictional claims in published maps and institutional affiliations.

\begin{abstract}
(c) (i) Open Access This article is licensed under a Creative Commons Attribution 4.0 International License, which permits use, sharing, adaptation, distribution and reproduction in any medium or format, as long as you give appropriate credit to the original author(s) and the source, provide a link to the Creative Commons license, and indicate if changes were made. The images or other third party material in this article are included in the article's Creative Commons license, unles indicated otherwise in a credit line to the material. If material is not included in the article's Creative Commons license and your intended use is not permitted by statutory regulation or exceeds the permitted use, you will need to obtain permission directly from the copyright holder. To view a copy of this license, visit http://creativecommons.org/ licenses/by/4.0/.
\end{abstract}

(C) The Author(s) 2021 